\title{
Removing the Faddeev-Popov zero modes from Yang-Mills theory in spacetimes with compact spatial sections
}

\author{
Jos Gibbons ${ }^{*}$ and Atsushi Higuch $\left.\right|^{\dagger}$ \\ Department of Mathematics, University of York, Heslington, York, YO10 5DD, United Kingdom
}

(Dated: January 7, 2015)

\begin{abstract}
It is well known that in de Sitter space the (free) minimally coupled massless scalar field theory does not admit any de Sitter-invariant Hadamard state. Related to this is the fact that the propagator for the massive scalar field corresponding to the de Sitter-invariant vacuum state diverges in the massless limit, with the infrared-divergent term being a constant. Since the Faddeev-Popov ghosts for the covariantly quantized Yang-Mills theory are minimally coupled massless scalar fields, it might appear that de Sitter symmetry would be broken in the ghost sector of Yang-Mills theory in de Sitter space. It is shown in this paper that the modes responsible for de Sitter symmetry breaking can be removed in a way consistent with BRST invariance and that a de Sitter-invariant theory can be constructed. More generally, it is shown that the spatially constant modes (the zero modes) of the Faddeev-Popov ghosts can be disposed of in a wide class of spacetimes with compact spatial sections. Then, the effective theory obtained by removing the zero modes, which contains a nonlocal interaction term, is shown to be equivalent to the theory corresponding to using a FaddeevPopov-ghost propagator with the constant infrared-divergent term removed, provided that one can freely integrate by parts in the spacetime integral at the vertex for the ghost interaction term.
\end{abstract}

PACS numbers: 04.62.+v, 11.10.Ef, 11.15.Bt, 03.70.+k

\section{INTRODUCTION}

Physics in de Sitter space has been studied extensively since inflationary cosmology was proposed in the early 1980s [1 5] because this spacetime is a very good approximation to the geometry of the Inflationary Universe. Recently the detection of remnants of the primordial gravitational waves was reported [6. If confirmed by further observations, this will provide strong evidence for an inflationary phase of our Universe and, hence, for physical relevance of de Sitter space. Moreover, the expansion of our Universe is believed to be accelerating [7, 8, and it may eventually expand exponentially, thus becoming approximately de Sitter space. The proposal of the dS/CFT correspondence [9] gives another motivation to study physics in de Sitter space. Thus, it will be useful to understand properties of Yang-Mills theory, which is an important ingredient of any realistic model of particle physics, in de Sitter space.

A method commonly used to quantize Yang-Mills theory is the canonical quantization that starts from the Lagrangian density with a covariant gauge-fixing term and the corresponding Faddeev-Popov (FP) term [10. In this method of quantization it can readily be seen that the FP ghosts, i.e. the FP ghost and antighost, are minimally coupled massless scalar fields. Now, it is well known that the (free) minimally coupled massless scalar field theory suffers from infrared (IR) divergences in de Sitter space [1] if one requires de Sitter invariance. As a result, there is no de Sitter-invariant Hadamard state for free minimally coupled massless scalar field theory [12.

\footnotetext{
* jg1047@york.ac.uk

$\dagger$ atsushi.higuchi@york.ac.uk
}

Thus, there appears to be no de Sitter-invariant perturbative vacuum state for the FP-ghost fields. If this were indeed the case, then de Sitter invariance would be broken via the FP-ghost sector in covariantly quantized Yang-Mills theory. In Ref. 13 it was pointed out that, if one regularizes the FP-ghost propagator by introducing a small mass, then the IR-divergent constant term in the propagator does not contribute to the amplitude because the FP ghosts interact with the gauge field through a derivative coupling. With this observation, it was proposed to use the effective IR-finite propagator defined by discarding the constant IR-divergent term and then taking the massless limit. This effective propagator is de Sitter-invariant. However, the mass term for the FP ghosts breaks BRST invariance, and it is not entirely clear whether BRST invariance is restored in the massless limit.

In this paper we present a new method to solve this IR problem in the FP-ghost sector in global de Sitter space, which has compact spatial sections. The resulting theory is compatible with BRST invariance of Yang-Mills theory. The crucial observation in our method is that the spatially constant modes causing IR divergences, which we call the zero modes, of the FP ghosts can be removed from the theory by requiring the physical states to be annihilated by certain conserved charges. We explain our method in a wider class of spacetimes with compact spatial sections to which global de Sitter space belongs. We also show that the theory we obtain is equivalent to that proposed in Ref. [13].

The rest of the paper is organized as follows. In Sec. II we review the incompatibility between de Sitter invariance and IR finiteness for the free minimally coupled massless scalar field. We then show that the zero modes can be removed from the theory by imposing the condi- 
tion that a certain conserved charge annihilate the physical states. In Sec. [II we discuss the conserved charges involving the FP ghosts that will be used to remove the zero modes. In particular, we discuss their relationship to the BRST and antiBRST charges. In Sec. IV] we write down the Hamiltonian of the theory that governs the physical states that are annihilated by the conserved charges discussed in Sec. III in a class of spacetimes that includes global de Sitter space. This effective Hamiltonian contains no zero modes but is coordinate dependent and has a nonlocal interaction term. In Sec. $\mathrm{V}$ we show that the theory described by the Hamiltonian found in Sec. IV is equivalent to that of Ref. [13] obtained by discarding the IR-divergent part of the FP-ghost propagator in the de Sitter case. Then we summarize and discuss our results in Sec. VI. In Appendix A we provide some technical details for Sec. III. In Appendix B we present the effective Hamiltonian of Sec. IV for the general spacetime with compact spatial sections. In Appendix C we justify the identification of the time component of the gauge field as (a multiple of) the conjugate momentum density for the Nakanishi-Lautrup auxiliary field by using the Dirac bracket. In Appendix D we discuss the redefinition of the inner product necessary for defining the states annihilated by the conserved charges of Sec. III] In Appendix E we illustrate some of our results in the special case of static torus space. Finally, in Appendix F we show that the perturbative vacuum state for the de Sitter case is automatically annihilated by the bosonic Noether charges discussed in Sec. III. We use natural units with $\hbar=c=1$ and the metric signature $+-\cdots-$ throughout this paper.

\section{MINIMALLY COUPLED MASSLESS SCALAR FIELD IN DE SITTER SPACE}

The problem we wish to discuss in this paper stems from the fact that the FP ghosts are minimally coupled massless scalar fields. For this reason we first briefly review some aspects of the IR problem for the (free) minimally coupled scalar field theory and the nonexistence of de Sitter-invariant Hadamard state for this theory [12]. We then show, as pointed out in Ref. [14, how a de Sitterinvariant state can be obtained in a new Hilbert space with a slightly modified inner product. (It is also known that there is a unitary representation of the de Sitter group corresponding to the minimally coupled massless scalar field [15.). This construction serves as a toy model for what we shall do for the FP-ghost sector of Yang-Mills theory.

The line element of $n$-dimensional de Sitter space in global coordinates is

$$
d s^{2}=d t^{2}-H^{-2} \cosh ^{2} H t d \Omega^{2},
$$

where $d \Omega^{2}$ is the line element of the $(n-1)$-dimensional unit sphere $S^{n-1}$ and where $H$ is a positive constant. We assume $n \geq 3$ here. Let $Y_{\ell \sigma}(\boldsymbol{\theta}), \ell=0,1,2, \ldots$, be the scalar spherical harmonics on $S^{n-1}$, where $\boldsymbol{\theta}=$ $\left(\theta_{1}, \theta_{2}, \ldots, \theta_{n-1}\right)$ are the angular coordinates on $S^{n-1}$ and where $\sigma$ represents all labels other than $\ell$. They satisfy

$$
\eta^{i j} \tilde{\nabla}_{i} \tilde{\nabla}_{j} Y_{\ell \sigma}(\boldsymbol{\theta})=-\ell(\ell+n-2) Y_{\ell \sigma}(\boldsymbol{\theta}),
$$

where $\tilde{\nabla}_{i}$ is the covariant derivative on $S^{n-1}$ with metric $\eta_{i j}$ and inverse metric $\eta^{i j}$. The spherical harmonics are normalized as

$$
\int_{S^{n-1}} d \Omega Y_{\ell \sigma}^{*}(\boldsymbol{\theta}) Y_{\ell^{\prime} \sigma^{\prime}}(\boldsymbol{\theta})=\delta_{\ell \ell^{\prime}} \delta_{\sigma \sigma^{\prime}}
$$

Then, the minimally coupled scalar field $\phi(t, \boldsymbol{\theta})$ of mass $M$ can be expanded as

$$
\phi(t, \boldsymbol{\theta})=\sum_{\ell=0}^{\infty} \sum_{\sigma}\left[a_{\ell \sigma} f_{\ell}(t) Y_{\ell \sigma}(\boldsymbol{\theta})+a_{\ell \sigma}^{\dagger} f_{\ell}^{*}(t) Y_{\ell \sigma}^{*}(\boldsymbol{\theta})\right] .
$$

The functions $f_{\ell}(t)$ are given by

$$
f_{\ell}(t)=H^{\frac{n-2}{2}} N_{\ell}(\cosh H t)^{-\frac{n-2}{2}} \mathrm{P}_{-\frac{1}{2}+\lambda}^{-\frac{n-2}{2}}(i \sinh H t),
$$

where

$$
\begin{aligned}
\lambda & =\sqrt{\left(\frac{n-1}{2}\right)^{2}-\left(\frac{M}{H}\right)^{2}}, \\
\left|N_{\ell}\right|^{2} & =\frac{\Gamma\left(\ell+\frac{n-1}{2}+\lambda\right) \Gamma\left(\ell+\frac{n-1}{2}-\lambda\right)}{2} .
\end{aligned}
$$

(See, e.g., Ref. 15.) The associated Legendre function is given by

$$
\begin{aligned}
\mathrm{P}_{\nu}^{-\mu}(x)= & \frac{1}{\Gamma(1+\mu)}\left(\frac{1-x}{1+x}\right)^{\frac{\mu}{2}} \\
& \times F\left(-\nu, \nu+1 ; 1+\mu ; \frac{1-x}{2}\right),
\end{aligned}
$$

where $F(\alpha, \beta ; \gamma ; z)$ is Gauss's hypergeometric function. The normalization constant $N_{\ell}$ in Eq. (7) has been determined by the requirement

$$
\begin{aligned}
{\left[a_{\ell \sigma}, a_{\ell^{\prime} \sigma^{\prime}}^{\dagger}\right] } & =\delta_{\ell \ell^{\prime}} \delta_{\sigma \sigma^{\prime}}, \\
{\left[a_{\ell \sigma}, a_{\ell^{\prime} \sigma^{\prime}}\right] } & =0,
\end{aligned}
$$

and the equal-time commutation relations of the field operator $\phi(t, \boldsymbol{\theta})$ and its time derivative. In particular,

$$
\left[\phi(t, \boldsymbol{\theta}), \dot{\phi}\left(t, \boldsymbol{\theta}^{\prime}\right)\right]=\frac{i H^{n-1}}{\cosh ^{n-1} H t} \delta\left(\boldsymbol{\theta}, \boldsymbol{\theta}^{\prime}\right) .
$$

Equation (5) can be rewritten by using

$$
F(\alpha, \beta ; \gamma ; z)=(1-z)^{\gamma-\alpha-\beta} F(\gamma-\alpha, \gamma-\beta ; \gamma ; z)
$$

as

$$
\begin{aligned}
f_{\ell}(t)= & \frac{H^{\frac{n-2}{2}} N_{\ell}(\cosh H t)^{\ell}}{2^{\ell+\frac{n-2}{2}} \Gamma\left(\ell+\frac{n}{2}\right)} \\
& \times F\left(b_{\ell+}, b_{\ell-} ; \ell+\frac{n}{2} ; \frac{1-i \sinh H t}{2}\right),
\end{aligned}
$$


where

$$
b_{\ell \pm}=\ell+\frac{n-1}{2} \pm \lambda .
$$

The de Sitter-invariant Bunch-Davies (or Euclidean) vacuum state $|0\rangle$ [16 [18] is defined by requiring $a_{\ell \sigma}|0\rangle=$ 0 for all $\ell$ and $\sigma$. The two-point Wightman function in this state is

$$
\left\langle 0\left|\phi(t, \boldsymbol{\theta}) \phi\left(t^{\prime}, \boldsymbol{\theta}^{\prime}\right)\right| 0\right\rangle=\sum_{\ell=0}^{\infty} f_{\ell}(t) f_{\ell}^{*}\left(t^{\prime}\right) \sum_{\sigma} Y_{\ell \sigma}(\boldsymbol{\theta}) Y_{\ell \sigma}^{*}\left(\boldsymbol{\theta}^{\prime}\right) .
$$

This two-point function is divergent as $M \rightarrow 0^{+}$because the space-independent mode function,

$$
F_{0}(t):=f_{0}(t) Y_{00}(\boldsymbol{\theta}),
$$

where $Y_{00}(\boldsymbol{\theta})$ is the space-independent spherical harmonic with $\ell=0$, is divergent in this limit. Hence, the two-point function given by Eq. 15 is divergent and the Bunch-Davies vacuum state will not be well defined in the massless limit.

In Appendix $\mathrm{A}$ it is shown that

$$
F_{0}(t)=\frac{1}{\sqrt{2 c_{0}}}\left\{\frac{1}{M}-M\left[g(t)+c_{1}+i c_{0} f(t)\right]\right\}+o(M),
$$

where

$$
\begin{aligned}
c_{0} & =\frac{\pi^{(n+1) / 2}}{\Gamma\left(\frac{n+1}{2}\right) H^{n}}, \\
f(t) & =\int_{0}^{t} \frac{d t^{\prime}}{V\left(t^{\prime}\right)}, \\
g(t) & =\int_{0}^{t} d t^{\prime}\left[\frac{1}{V\left(t^{\prime}\right)} \int_{0}^{t^{\prime}} d t^{\prime \prime} V\left(t^{\prime \prime}\right)\right] .
\end{aligned}
$$

Here,

$$
V(t):=\frac{2 \pi^{n / 2}}{\Gamma\left(\frac{n}{2}\right) H^{n-1}} \cosh ^{n-1} H t
$$

is the volume of the spatial section at time $t$, which is an $(n-1)$-dimensional sphere of radius $H^{-1} \cosh H t$. Note that

$$
V_{S^{n-1}}=\frac{2 \pi^{n / 2}}{\Gamma\left(\frac{n}{2}\right)}
$$

is the volume of the unit $S^{n-1}$. We do not need the value of the constant $c_{1}$, which is given in Eq. A16) for completeness.

Although there is no de Sitter-invariant Bunch-Davies vacuum for the minimally coupled massless scalar field, there are $O(n)$-invariant states [12, 19]. To see this, we note first that the $\ell=0$ sector of the field $\phi(t, \boldsymbol{\theta})$, i.e. the space-independent sector, which we denote by $\phi_{(0)}(t)$ and call the zero mode, satisfies

$$
\frac{d}{d t}\left[V(t) \frac{d \phi_{(0)}(t)}{d t}\right]=0,
$$

with the general solution

$$
\phi_{(0)}(t)=\hat{q}+\hat{p} f(t),
$$

where $f(t)$ is defined by Eq. (19). Thus, the massless field $\phi(t, \boldsymbol{\theta})$ can be written, with the zero mode separated out, as

$$
\begin{aligned}
\phi(t, \boldsymbol{\theta})= & \hat{q}+\hat{p} f(t) \\
& +\sum_{\ell=1}^{\infty} \sum_{\sigma}\left[a_{\ell \sigma} f_{\ell}(t) Y_{\ell \sigma}(\boldsymbol{\theta})+a_{\ell \sigma}^{\dagger} f_{\ell}^{*}(t) Y_{\ell \sigma}^{*}(\boldsymbol{\theta})\right] .
\end{aligned}
$$

By integrating the equal-time commutator 11 over the space we find

$$
[\hat{q}, \hat{p}]=i,
$$

which is identical with the commutator between the position and momentum operators in one-dimensional quantum mechanics. Thus, one can represent the zeromode sector by a normalized wave function $\psi(q)$ and let $\hat{p}=-i d / d q$. If we define $\left|0_{(+)}\right\rangle$to be the vacuum state for the $\ell \neq 0$ sector satisfying the requirement $a_{\ell \sigma}\left|0_{(+)}\right\rangle=0$ for all $\ell>0$ and $\sigma$, then the state $|\psi\rangle=\psi(q) \otimes\left|0_{(+)}\right\rangle$is $O(n)$ invariant but not de Sitter-invariant.

To show that the state $|\psi\rangle$ is not de Sitter-invariant, we examine the action of a de Sitter boost on the operators $\hat{q}, \hat{p}$ and $a_{\ell \sigma}$. Let us parametrize the unit $S^{n-1}$ using the natural embedding space $\mathbb{R}^{n}$ as $x_{1}=\cos \chi, x_{2}=\tilde{x}_{2} \sin \chi$, $\ldots, x_{n}=\tilde{x}_{n} \sin \chi$, where $\chi \in[0, \pi]$ and $\tilde{x}_{2}^{2}+\cdots+\tilde{x}_{n}^{2}=1$. Let $\tilde{Y}_{m \tilde{\sigma}}$ be the spherical harmonics on the unit $S^{n-2}$ with eigenvalues $-m(m+n-3), m=0,1,2, \ldots$, of the Laplacian there. Then the spherical harmonics $Y_{\ell m \tilde{\sigma}}$ on $S^{n-1}$ proportional to $\tilde{Y}_{m \tilde{\sigma}}$ are given by [15]

$$
Y_{\ell m \tilde{\sigma}}(\boldsymbol{\theta})=c_{\ell m}(\sin \chi)^{-(n-3) / 2} \mathrm{P}_{\ell+(n-3) / 2}^{-(m+(n-3) / 2)}(\cos \chi) \tilde{Y}_{m \tilde{\sigma}},
$$

where

$$
c_{\ell m}=\left[\frac{2 \ell+n-2}{2} \cdot \frac{(\ell+m+n-2) !}{(\ell-m) !}\right]^{1 / 2} .
$$

It is clear that the mode functions given by

$$
F_{\ell m \tilde{\sigma}}(t, \boldsymbol{\theta})=f_{\ell}(t) Y_{\ell m \tilde{\sigma}}(\boldsymbol{\theta})
$$

transform to one another, i.e. they do not transform into $F_{\ell m \tilde{\sigma}}^{*}(t, \boldsymbol{\theta})$, under the $O(n)$ transformations. If $M>0$, it can be shown that the functions $F_{\ell m \tilde{\sigma}}(t, \boldsymbol{\theta})$ transform among themselves under de Sitter boosts as well [15]. To show this, it is sufficient to examine the action of the boost Killing vector in the $x_{1}$ direction given by

$$
L_{X}:=\frac{\cos \chi}{H} \frac{\partial}{\partial t}-\tanh H t \sin \chi \frac{\partial}{\partial \chi},
$$

because the component connected to the identity of the de Sitter group is generated by $L_{X}$ and the $S O(n)$ rotations. Indeed we show in Appendix A that

$$
\begin{aligned}
L_{X} F_{\ell m \tilde{\sigma}}(t, \boldsymbol{\theta})= & -i k_{\ell m} F_{(\ell-1) m \tilde{\sigma}}(t, \boldsymbol{\theta}) \\
& -i k_{(\ell+1) m} F_{(\ell+1) m \tilde{\sigma}}(t, \boldsymbol{\theta}),
\end{aligned}
$$


where

$$
\begin{aligned}
k_{\ell m}= & {\left[\frac{(\ell-m)(\ell+m+n-3)}{(2 \ell+n-2)(2 \ell+n-4)}\right]^{1 / 2} } \\
& \times\left[(\ell-1)(\ell+n-2)+\frac{M^{2}}{H^{2}}\right]^{1 / 2} .
\end{aligned}
$$

The transformation (31) is unaltered for the massless case if $\ell \geq 2$ or $(\ell, m)=(1,1)$, but for $(\ell, m)=(0,0)$ and $(\ell, m)=(1,0)$ it needs to be reexamined because the $\ell=0$ mode function $F_{0}(t)$ is divergent in the massless limit. Using Eq. (17) in Eq. (31) with $\ell=1$ and $m=0$, we obtain

$$
L_{X} F_{10}(t, \boldsymbol{\theta})=-\frac{i}{\sqrt{2 n c_{0} H^{2}}}-i k_{20} F_{20}(t, \boldsymbol{\theta}) .
$$

We have omitted the label $\tilde{\sigma}$ for $m=0$ because there is only one independent spherical harmonic for each $\ell$ with $m=0$. Next we observe that

$$
\begin{aligned}
L_{X} f(t) & =-\sqrt{\frac{2}{c_{0}}} \lim _{M \rightarrow 0^{+}} M^{-1} \operatorname{Im}\left[L_{X} F_{0}(t)\right] \\
& =\frac{1}{\sqrt{2 n c_{0} H^{2}}}\left[F_{10}(t, \boldsymbol{\theta})+F_{10}^{*}(t, \boldsymbol{\theta})\right] .
\end{aligned}
$$

The boost $L_{X}$ acts on the field $\phi(t, \boldsymbol{\theta})$ given by Eq. (25) as

$$
\begin{aligned}
& L_{X} \phi(t, \boldsymbol{\theta}) \\
& =\hat{p} L_{X} f(t) \\
& +\sum_{\ell=1}^{\infty} \sum_{m=0}^{\ell} \sum_{\tilde{\sigma}}\left[a_{\ell m \tilde{\sigma}} L_{X} F_{\ell m \tilde{\sigma}}(t, \boldsymbol{\theta})+a_{\ell m \tilde{\sigma}}^{\dagger} L_{X} F_{\ell m \tilde{\sigma}}^{*}(t, \boldsymbol{\theta})\right] .
\end{aligned}
$$

We rewrite this boost transformation of $\phi(t, \boldsymbol{\theta})$ in such a way that the transformation is attributed to the operators:

$$
\begin{aligned}
& L_{X} \phi(t, \boldsymbol{\theta}) \\
& =\delta_{X} \hat{q}+f(t) \delta_{X} \hat{p} \\
& +\sum_{\ell=1}^{\infty} \sum_{m=0}^{\ell} \sum_{\tilde{\sigma}}\left[F_{\ell m \tilde{\sigma}}(t, \boldsymbol{\theta}) \delta_{X} a_{\ell m \tilde{\sigma}}+F_{\ell m \tilde{\sigma}}^{*}(t, \boldsymbol{\theta}) \delta_{X} a_{\ell m \tilde{\sigma}}^{\dagger}\right] .
\end{aligned}
$$

Thus, for $\ell \geq 2$ and $(\ell, m)=(1,1)$ we find

$$
\delta_{X} a_{\ell m \tilde{\sigma}}=-i k_{\ell m} a_{(\ell-1) m \tilde{\sigma}}-i k_{(\ell+1) m} a_{(\ell+1) m \tilde{\sigma}} .
$$

For the other operators we have

$$
\begin{aligned}
\delta_{X} \hat{q} & =\frac{i}{\sqrt{2 n c_{0} H^{2}}}\left(a_{10}^{\dagger}-a_{10}\right), \\
\delta_{X} \hat{p} & =0, \\
\delta_{X} a_{10} & =\frac{1}{\sqrt{2 n c_{0} H^{2}}} \hat{p}-i k_{20} a_{20} .
\end{aligned}
$$

(The invariance of $\hat{p}$ under de Sitter transformations can also be inferred by noting that $\hat{p}$ is the Noether charge corresponding to the conserved current $\nabla^{\mu} \phi$ of the theory.)

Equation 40 implies that the conditions $a_{\ell m \tilde{\sigma}}|\psi\rangle=0$ are not de Sitter-invariant unless the condition $\hat{p}|\psi\rangle=0$ is also imposed. Conversely, Eq. (39) implies that these conditions taken together are de Sitter-invariant. Thus, the vacuum state $|\psi\rangle$ defined by requiring $a_{\ell \sigma}|\psi\rangle=0$ and $\hat{p}|\psi\rangle=0$ is de Sitter-invariant. However, the condition $\hat{p}|\psi\rangle=-i \psi^{\prime}(q) \otimes\left|0_{(+)}\right\rangle=0$ implies that $\psi(q)$ is a constant function. Then $\int d q|\psi(q)|^{2}=\infty$. Therefore, there is no normalizable de Sitter-invariant state of the form $\psi(q) \otimes\left|0_{(+)}\right\rangle$.

However, it is possible to redefine the inner product of states $\left|\Psi_{1}\right\rangle=\psi(q) \otimes\left|\alpha_{1}\right\rangle$ and $\left|\Psi_{2}\right\rangle=\psi(q) \otimes\left|\alpha_{2}\right\rangle$, where $\left|\alpha_{1}\right\rangle$ and $\left|\alpha_{2}\right\rangle$ are states in the Fock space built by applying creation operators $a_{\ell \sigma}$ on $\left|0_{(+)}\right\rangle$and where $\psi(q)$ is constant, simply as $\left\langle\Psi_{1} \mid \Psi_{2}\right\rangle=\left\langle\alpha_{1} \mid \alpha_{2}\right\rangle$. The state $\psi(q) \otimes\left|0_{(+)}\right\rangle$with $\psi(q)=$ const. is a well-defined de Sitter-invariant state with this inner product. A similar redefinition of the inner product was used in quantum cosmology [20]. This redefinition is closely related to the method of "group averaging" (see, e.g. Ref. [21 23]), which has been incorporated into the refined algebraic quantization 24].

If the operators $\hat{q}$ and $\hat{p}$ are physical observables, this redefinition of inner product described above will not be physical because, e.g. the expectation value of $\hat{q}$ will be undefined. However, since the FP ghosts are not physical particles, a similar redefinition of inner product for these fields will not affect true physical quantities. In the next section we identify conserved charges analogous to the operator $\hat{p}$ that can be used to 'banish' the zero modes of the FP ghosts.

\section{SOME CONSERVED CHARGES IN COVARIANTLY QUANTIZED YANG-MILLS THEORY}

In this section we examine covariantly quantized YangMills theory in the Landau gauge in (globally hyperbolic) spacetime with compact spatial sections. Let $f^{a b c}$ be the totally antisymmetric structure constant for a compact semisimple Lie group. Thus, if $T^{a}$ are the generators of this group, then

$$
\left[T^{a}, T^{b}\right]=i f^{a b c} T^{c}
$$

where the repeated Lie-algebra indices are summed over. The fields in Yang-Mills theory are the gauge field $A_{\mu}^{a}$ and the FP-ghost and antighost fields, $c^{a}$ and $\bar{c}^{a}$. It is possible to introduce other gauge multiplets, but we do not do so in this paper. One defines the field strength as $F_{\mu \nu}^{a}=\nabla_{\mu} A_{\nu}^{a}-\nabla_{\nu} A_{\mu}^{a}+q f^{a b c} A_{\mu}^{b} A_{\nu}^{c}$, where $q$ is the coupling constant. We introduce the following notation [26]:

$$
\begin{aligned}
X \cdot Y & :=X^{a} Y^{a}, \\
(X \times Y)^{a} & :=f^{a b c} X^{b} X^{c} .
\end{aligned}
$$


In this simplified notation the field strength is given as

$$
F_{\mu \nu}=\nabla_{\mu} A_{\nu}-\nabla_{\nu} A_{\mu}+q A_{\mu} \times A_{\nu}
$$

The Lagrangian density is given by

$$
\begin{gathered}
\mathcal{L}=\sqrt{|g|}\left\{-\frac{1}{4} F_{\mu \nu} \cdot F^{\mu \nu}-i \nabla^{\mu} \bar{c} \cdot D_{\mu} c\right. \\
\left.-\frac{1}{2 \xi} \nabla_{\mu} A^{\mu} \cdot \nabla_{\nu} A^{\nu}\right\}
\end{gathered}
$$

where

$$
D_{\mu} c=\nabla_{\mu} c+q A_{\mu} \times c .
$$

Here, $g$ is the determinant of the background spacetime metric $g_{\mu \nu}$, and the FP-ghost field $c^{a}$ and antighost field $\bar{c}^{a}$ are fermionic Hermitian fields [25, 26. This Lagrangian density can be rewritten by introducing the Nakanishi-Lautrup auxiliary field $B^{a}$ [27, 28] as

$$
\begin{aligned}
\mathcal{L}= & \sqrt{|g|}\left\{-\frac{1}{4} F_{\mu \nu} \cdot F^{\mu \nu}-i \nabla^{\mu} \bar{c} \cdot D_{\mu} c\right. \\
& \left.-\nabla_{\mu} B \cdot A^{\mu}+\frac{\xi}{2} B \cdot B\right\} .
\end{aligned}
$$

One can eliminate the field $B^{a}$ using its field equation from this Lagrangian density and show that it is equivalent to the Lagrangian density in Eq. 45 up to a total derivative.

The Lagrangian density (47) is invariant (up to a total derivative) under the BRST transformation [29, 30]:

$$
\begin{aligned}
\delta_{B} A_{\mu} & =\epsilon D_{\mu} c, \\
\delta_{B} c & =-\frac{1}{2} \epsilon q c \times c, \\
\delta_{B} \bar{c} & =i \epsilon B, \\
\delta_{B} B & =0,
\end{aligned}
$$

where $\epsilon$ is a Grassmann number anticommuting with $c$ and $\bar{c}$. The conserved current corresponding to this invariance can be given as 26]

$$
J_{B}^{\mu}=B \cdot D^{\mu} c-\nabla^{\mu} B \cdot c+\frac{i}{2} q \nabla^{\mu} \bar{c} \cdot(c \times c)-\nabla_{\nu}\left(F^{\mu \nu} \cdot c\right) .
$$

The conserved BRST charge is

$$
Q_{B}=\int_{\Sigma} d \Sigma_{\mu} J_{B}^{\mu}
$$

where $\Sigma$ is a Cauchy surface. In this paper we specialize to the Landau gauge $\xi=0$. Then the Lagrangian density 47 is

$$
\mathcal{L}_{\text {Lan }}=\sqrt{|g|}\left\{-\frac{1}{4} F_{\mu \nu} \cdot F^{\mu \nu}-i \nabla^{\mu} \bar{c} \cdot D_{\mu} c-\nabla_{\mu} B \cdot A^{\mu}\right\} .
$$

The FP and gauge-fixing terms in the action can be rewritten as follows:

$$
\begin{aligned}
& \int d x \sqrt{|g|}\left\{-i \nabla^{\mu} \bar{c} \cdot D_{\mu} c-\nabla_{\mu} B \cdot A^{\mu}\right\} \\
& =\int d x \sqrt{|g|}\left\{i \nabla^{\mu} c \cdot D_{\mu} \bar{c}-\nabla_{\mu}(B-i q \bar{c} \times c) \cdot A^{\mu}\right\},
\end{aligned}
$$

where the integral is over the spacetime and where the surface terms have been dropped. The second form of this part of the action can be obtained from the first by interchanging $c$ and $\bar{c}$ and changing $B$ to $B-i q \bar{c} \times c$. This makes it clear that the Lagrangian density $\mathcal{L}_{\text {Lan }}$ is also invariant (up to a total derivative) under the following transformation called the antiBRST transformation [3133.

$$
\begin{aligned}
\delta_{\bar{B}} A_{\mu} & =\epsilon D_{\mu} \bar{c}, \\
\delta_{\bar{B}} \bar{c} & =-\frac{1}{2} \epsilon q \bar{c} \times \bar{c}, \\
\delta_{\bar{B}} c & =-i \epsilon(B-i q \bar{c} \times c), \\
\delta_{\bar{B}} B & =-q \epsilon \bar{c} \times B .
\end{aligned}
$$

(In fact the antiBRST transformation can be defined for all values of $\xi 32$.)

Now, the Euler-Lagrange equations of motion arising from the variations of $B, c$ and $\bar{c}$ are

$$
\begin{aligned}
\nabla_{\mu} A^{\mu} & =0, \\
\nabla_{\mu} D^{\mu} c & =0, \\
\nabla_{\mu} D^{\mu} \bar{c} & =0 .
\end{aligned}
$$

One needs to use Eq. 60 to derive Eq. 62 from the original Euler-Lagrange equation. If we let $q=0$ in the last two equations, we find $\nabla_{\mu} \nabla^{\mu} c=\nabla_{\mu} \nabla^{\mu} \bar{c}=0$. That is, the FP ghost and antighost are minimally coupled massless scalar fields.

Eqs. 60- 62 imply that the following charges are conserved:

$$
\begin{aligned}
Q_{A} & =\int_{\Sigma} d \Sigma_{\mu} A^{\mu} \\
Q_{D c} & =\int_{\Sigma} d \Sigma_{\mu} D^{\mu} c \\
Q_{D \bar{c}} & =\int_{\Sigma} d \Sigma_{\mu} D^{\mu} \bar{c}
\end{aligned}
$$

where $\Sigma$ is a Cauchy surface. (Note that these charges carry a Lie-algebra index.) These are the Noether charges corresponding to symmetries of the Lagrangian, as can readily be verified. Our proposal is to require that the physical states be annihilated by these charges. Thus, we impose the following conditions on the physical states $\mid$ phys $\rangle$ as well as the usual condition $Q_{B} \mid$ phys $\rangle=0$ :

$$
\left.\left.\left.Q_{A} \mid \text { phys }\right\rangle=Q_{D c} \mid \text { phys }\right\rangle=Q_{D \bar{c}} \mid \text { phys }\right\rangle=0 .
$$

The symmetries these charges generate are spacetime scalars. Therefore, they commute with spacetime symmetry generators. As a result, the conditions 66 are invariant under any continuous spacetime symmetries. As we shall see in the next section, these conditions eliminate the spatially constant modes from FP ghosts. For the de Sitter case this will lead to de Sitter-invariant perturbation theory.

In fact, the condition $Q_{D c} \mid$ phys $\rangle=0$ is a consequence of the condition $Q_{A} \mid$ phys $\rangle=0$ and the BRST invariance of the physical states because $\left[Q_{B}, Q_{A}\right]=-i Q_{D c}[26]$. If 
we require antiBRST invariance, with the corresponding conserved charge $Q_{\bar{B}}$, of the physical states as well, then the condition $Q_{D \bar{c}} \mid$ phys $\rangle=0$ will also be a consequence of the condition $Q_{A} \mid$ phys $\rangle=0$ because $\left[Q_{\bar{B}}, Q_{A}\right]=$ $-i Q_{D \bar{c}}$. These observations naturally lead to the observation that one also needs to impose the condition $\left\{Q_{B}, Q_{D \bar{c}}\right\} \mid$ phys $\rangle=0$. (Note that $\left\{Q_{B}, Q_{D c}\right\}=0$ because of the nilpotency of $Q_{B}$, i.e. $Q_{B}^{2}=0$.) This condition turns out to have a natural interpretation. The field equation for $A_{\mu}$ can be written as 32, 34.

$$
\nabla^{\nu} F_{\nu \mu}+q J_{\mu}=\left\{Q_{B}, D_{\mu} \bar{c}\right\}=-\left\{Q_{\bar{B}}, D_{\mu} c\right\},
$$

where $J^{\mu}$ is the Noether current for the global gauge transformation [26]:

$$
J^{\mu}:=A_{\nu} \times F^{\nu \mu}+A^{\mu} \times B-i \bar{c} \times D^{\mu} c+i \nabla^{\mu} \bar{c} \times c .
$$

The corresponding Noether charge is

$$
Q_{\mathrm{gg}}=\int_{\Sigma} d \Sigma_{\mu} J^{\mu}
$$

By integrating Eq. 67) over a (compact) Cauchy surface, we obtain

$$
q Q_{\mathrm{gg}}=\left\{Q_{B}, Q_{D \bar{c}}\right\}=-\left\{Q_{\bar{B}}, Q_{D c}\right\} .
$$

Thus, the condition $Q_{\mathrm{gg}}|\mathrm{phys}\rangle=0$ on the physical states results from the condition $Q_{D \bar{c}}|\mathrm{phys}\rangle=0$ and the BRST invariance of the physical states. It will also result from the condition $Q_{D c} \mid$ phys $\rangle=0$ if we require the antiBRST invariance of the physical states.

The nilpotency of the BRST and antiBRST charges imply that $\left\{Q_{B}, Q_{\mathrm{gg}}\right\}=\left\{Q_{\bar{B}}, Q_{\mathrm{gg}}\right\}=0$. Thus, the conditions given by Eq. 666 are compatible with the BRST and antiBRST invariance if we also impose the condition $Q_{\text {gg }} \mid$ phys $\rangle=0$, i.e. the requirement that the physical state be invariant under the global gauge transformations. No more conditions are required for consistency of our conditions (66) with BRST or antiBRST invariance. (One can show that $\left\{Q_{B}, Q_{\bar{B}}\right\}=0$ as is well known and that $\left\{Q_{D c}^{a}, Q_{D \bar{c}}^{b}\right\}=q f^{a b c} Q_{A}^{c}$.) In the next section we show that the conditions (66) lead to an effective Hamiltonian that does not depend on the zero modes of the FP ghosts.

\section{THE HAMILTONIAN WITHOUT ZERO MODES}

In this section we analyze the FP ghosts of Yang-Mills theory in spacetime with compact spatial sections with the following line element:

$$
d s^{2}=[N(\mathbf{x})]^{2} d t^{2}-\gamma_{i j}(t, \mathbf{x}) d x^{i} d x^{j},
$$

where $\mathbf{x}=\left(x_{1}, x_{2}, \ldots, x_{n-1}\right)$. We also assume that the determinant of the spatial metric factorizes as

$$
\gamma(t, \mathbf{x})=h_{1}(t) h_{2}(\mathbf{x}) .
$$

The standard metric of global de Sitter space satisfies this property. We shall show that the conditions (66) lead to a Hamiltonian without zero modes. We discuss the Hamiltonian with a line element of a more general form in Appendix B.

The Lagrangian is obtained by integrating the Lagrangian density (54) over a Cauchy surface of constant time as

$$
L=\int d \mathbf{x} \mathcal{L}_{\text {Lan }}
$$

The conjugate momentum densities for the fields $A_{i}, B$, $c$ and $\bar{c}$ can be found as follows:

$$
\begin{aligned}
& \pi_{A}^{i}(t, \mathbf{x})=\frac{\delta L}{\delta \dot{A}_{i}(t, \mathbf{x})}=-\sqrt{|g|} F^{0 i}(t, \mathbf{x}), \\
& \pi_{B}(t, \mathbf{x})=\frac{\delta L}{\delta \dot{B}(t, \mathbf{x})}=-\sqrt{|g|} A^{0}(t, \mathbf{x}), \\
& \pi_{c}(t, \mathbf{x})=\frac{\delta L}{\delta \dot{c}(t, \mathbf{x})}=i \sqrt{|g|} \nabla^{0} \bar{c}(t, \mathbf{x}), \\
& \pi_{\bar{c}}(t, \mathbf{x})=\frac{\delta L}{\delta \dot{\bar{c}}(t, \mathbf{x})}=-i \sqrt{|g|} D^{0} c(t, \mathbf{x}),
\end{aligned}
$$

where the functional derivative with respect to a fermionic variable, e.g. $\delta L / \delta \dot{c}(t, \mathbf{x})$, is taken from the left, and where the indices are raised by the full inverse metric $g^{\mu \nu}$. Equation 75 is a second-class constraint. We show in Appendix C that the quantization using the Dirac bracket to deal with this constraint equation is equivalent to regarding it as defining $-\sqrt{|g|} A^{0}:=\pi_{B}$ without treating $A_{0}^{a}$ as independent dynamical variables.

The Hamiltonian density can readily be found as follows:

$$
\begin{aligned}
\mathcal{H} & =\dot{A}_{i} \cdot \pi_{A}^{i}+\dot{B} \cdot \pi_{B}+\dot{c} \cdot \pi_{c}+\dot{\bar{c}} \cdot \pi_{\bar{c}}-\mathcal{L}_{\mathrm{Lan}} \\
& =\mathcal{H}_{\text {class }}+\mathcal{H}_{\mathrm{GF}+\mathrm{FP}}
\end{aligned}
$$

where the classical contribution to the Hamiltonian density is

$$
\begin{aligned}
\mathcal{H}_{\text {class }}= & \frac{N}{2 \sqrt{\gamma}} \gamma_{i j} \pi^{i} \cdot \pi^{j}+\frac{\sqrt{\gamma} N}{4} \gamma^{i j} \gamma^{m n} F_{i m} \cdot F_{j n} \\
& +D_{i} A_{0} \cdot \pi^{i},
\end{aligned}
$$

with $A_{0}=-N \pi_{B} / \sqrt{\gamma}$, and where the contribution from the gauge-fixing and FP terms is

$$
\begin{aligned}
\mathcal{H}_{\mathrm{GF}+\mathrm{FP}}= & -\frac{N}{\sqrt{\gamma}} \pi_{c} \cdot\left(i \pi_{\bar{c}}+q \pi_{B} \times c\right) \\
& -N \sqrt{\gamma} \gamma^{i j}\left(i \nabla_{i} \bar{c} \cdot D_{j} c+\nabla_{i} B \cdot A_{j}\right) \\
= & -i \frac{\sqrt{\gamma}}{N} \nabla_{0} \bar{c} \cdot \nabla_{0} c \\
& -N \sqrt{\gamma} \gamma^{i j}\left(i \nabla_{i} \bar{c} \cdot D_{j} c+\nabla_{i} B \cdot A_{j}\right) .
\end{aligned}
$$

Here, the tensor $\gamma^{i j}$ is the inverse of $\gamma_{i j}$ as a matrix.

Now, let us define the zero mode for the field $c$ by

$$
c_{(0)}(t):=\frac{1}{V(t)} \int d \mathbf{x} \frac{\sqrt{\gamma}}{N} c(t, \mathbf{x}),
$$


where

$$
V(t):=\int d \mathbf{x} \frac{\sqrt{\gamma}}{N} .
$$

Let us also define $c_{(+)}:=c-c_{(0)}$. It is clear that

$$
\int d \mathbf{x} \frac{\sqrt{\gamma}}{N} c_{(+)}(t, \mathbf{x})=0
$$

We define $\bar{c}_{(0)}$ and $\bar{c}_{(+)}$from the field $\bar{c}$ in the same way. Our aim is to construct a Hamiltonian that does not depend on the zero modes $c_{(0)}$ and $\bar{c}_{(0)}$ after imposing the conditions (66).

The Hamiltonian density $\mathcal{H}_{\mathrm{GF}+\mathrm{FP}}$ given by Eq. 80 depends on $c_{(0)}$ as it stands. However, this dependence can be eliminated by the following redefinition of the Nakanishi-Lautrup auxiliary field:

$$
\tilde{B}:=B-i q \bar{c} \times c_{(0)} .
$$

We define the fields $\tilde{B}_{(0)}$ and $\tilde{B}_{(+)}$for $\tilde{B}$ in the same way as those for $c$ and $\bar{c}$. By substituting Eq. (85) into the Lagrangian density (54) we find that the new canonical conjugate momentum densities of $\tilde{B}, c$ and $\bar{c}$ are

$$
\begin{aligned}
\Pi_{\tilde{B}} & =\pi_{\tilde{B}}=-\sqrt{|g|} A^{0}=\frac{\sqrt{\gamma}}{N} \varpi_{\tilde{B}}, \\
\Pi_{c} & =\frac{\sqrt{\gamma}}{N} \varpi_{c}, \\
\Pi_{\bar{c}} & =\frac{\sqrt{\gamma}}{N} \varpi_{\bar{c}},
\end{aligned}
$$

where

$$
\begin{aligned}
\varpi_{\tilde{B}} & :=-A_{0}, \\
\varpi_{c} & :=i\left[\nabla_{0} \bar{c}-\frac{q}{V} \int d \mathbf{x} \frac{\sqrt{\gamma}}{N} \varpi_{\tilde{B}} \times \bar{c}\right], \\
\varpi_{\bar{c}} & :=-i\left[\nabla_{0} c-q \varpi_{\tilde{B}} \times c_{(+)}\right] .
\end{aligned}
$$

We define the zero modes $\varpi_{\tilde{B}(0)}$ of $\varpi_{\tilde{B}}$ as

$$
\varpi_{\tilde{B}(0)}(t):=\frac{1}{V} \int d \mathbf{x} \frac{\sqrt{\gamma}}{N} \varpi_{\tilde{B}}(t, \mathbf{x}) .
$$

The zero modes $\varpi_{c(0)}$ and $\varpi_{\bar{c}(0)}$ are defined in exactly the same way from $\varpi_{c}$ and $\varpi_{\bar{c}}$. We also define

$$
\varpi_{X(+)}:=\varpi_{X}-\varpi_{X(0)}, \quad X=\tilde{B}, c, \bar{c} .
$$

Then the equal-time canonical (anti)commutation relations, $\left[\Pi_{X}\left(t, \mathbf{x}^{\prime}\right), X(t, \mathbf{x})\right]_{ \pm}=-i \delta\left(\mathbf{x}, \mathbf{x}^{\prime}\right)$, where $[\ldots, \ldots]_{ \pm}$ is the commutator for $X=\tilde{B}$ and anticommutator for $X=c$ and $\bar{c}$, lead to

$$
\begin{aligned}
{\left[\varpi_{X(0)}(t), X_{(0)}(t)\right]_{ \pm} } & =-\frac{i}{V}, \\
{\left[\varpi_{X(+)}(t, \mathbf{x}), X_{(+)}\left(t, \mathbf{x}^{\prime}\right)\right]_{ \pm} } & =-i \frac{N}{\sqrt{\gamma}} \delta\left(\mathbf{x}, \mathbf{x}^{\prime}\right)+\frac{i}{V}, \\
{\left.\left[\varpi_{X(+)}(t, \mathbf{x}), X_{(0)}(t)\right)\right]_{ \pm} } & =\left[\varpi_{X(0)}(t), X_{(+)}\left(t, \mathbf{x}^{\prime}\right)\right]_{ \pm}=0 .
\end{aligned}
$$

Thus, the variables $V \varpi_{\tilde{B}(0)}, V \varpi_{c(0)}$ and $V \varpi_{\bar{c}(0)}$ are the canonical conjugate momenta of the zero modes $\tilde{B}_{(0)}$, $c_{(0)}$ and $\bar{c}_{(0)}$, respectively. Therefore, in the functional Schrödinger representation, where states are represented as functionals of $B(\mathbf{x}), c(\mathbf{x})$ and $\bar{c}(\mathbf{x})$, these operators are expressed as

$$
V \varpi_{X(0)}=-i \frac{\partial}{\partial X_{(0)}}, \quad X=\tilde{B}, c, \bar{c} .
$$

Now, we have

$$
\begin{aligned}
V \varpi_{\tilde{B}(0)} & =-Q_{A}, \\
V \varpi_{c(0)} & =i Q_{D \bar{c}}, \\
V \varpi_{\bar{c}(0)} & =-i\left[Q_{D c}-q c_{(0)} \times Q_{A}\right] .
\end{aligned}
$$

Thus, the conditions (66) are equivalent to

$$
\left.\left.\left.\varpi_{\tilde{B}(0)} \mid \text { phys }\right\rangle=\varpi_{c(0)} \mid \text { phys }\right\rangle=\varpi_{\bar{c}(0)} \mid \text { phys }\right\rangle=0 .
$$

These conditions imply that a state represented as a wave functional of $\tilde{B}, c$ and $\bar{c}$ does not depend on the zero modes, $\tilde{B}_{(0)}, c_{(0)}$ or $\bar{c}_{(0)}$. Thus, by requiring these conditions for the physical states, we can eliminate the FP zero modes. (It is necessary to redefine the inner product among the physical states. This point is discussed in Appendix D)

Next, we find the Hamiltonian obtained by eliminating the zero modes of $\tilde{B}, c$ and $\bar{c}$ in this manner. The Hamiltonian written in terms of the field variables and their time derivatives (rather than their canonical conjugate momentum densities) is invariant under the field redefinition (85) provided that the metric is of the form (71) with the property 72 as shown in Appendix B. Thus, one can solve Eqs. (90) and 91) for $\nabla_{0} c$ and $\nabla_{0} \bar{c}$ and substitute the resulting expressions into Eq. 81) to find the Hamiltonian. Then, we find the effective Hamiltonian $H_{\text {eff }}$ applicable to the physical states satisfying the conditions (101) by letting $\varpi_{X(0)}=0$, i.e. $\varpi_{X}=\varpi_{X(+)}$, $X=\tilde{B}, c, \bar{c}$. The result is

$$
H_{\mathrm{eff}}=\int d \mathbf{x}\left(\mathcal{H}_{\text {class }}+\mathcal{H}_{\mathrm{GF}+\mathrm{FP}}\right)+H_{\mathrm{GF}+\mathrm{FP}}^{\prime},
$$

where

$$
\begin{aligned}
\mathcal{H}_{\mathrm{GF}+\mathrm{FP}}= & \frac{\sqrt{\gamma}}{N} \varpi_{c(+)} \cdot\left(i \varpi_{\bar{c}(+)}+q \varpi_{\tilde{B}(+)} \times c_{(+)}\right) \\
& -N \sqrt{\gamma} \gamma^{i j}\left(i \nabla_{i} \bar{c}_{(+)} \cdot D_{j} c_{(+)}+\nabla_{i} \tilde{B}_{(+)} \cdot A_{j}\right)
\end{aligned}
$$

and

$$
H_{\mathrm{GF}+\mathrm{FP}}^{\prime}=-\frac{i q^{2}}{V} \bar{F} \cdot F
$$

with

$$
\begin{aligned}
& F:=\int d \mathbf{x} \frac{\sqrt{\gamma}}{N}\left(\varpi_{\tilde{B}(+)} \times \bar{c}_{(+)}\right), \\
& \bar{F}:=\int d \mathbf{x} \frac{\sqrt{\gamma}}{N}\left(\varpi_{\tilde{B}(+)} \times c_{(+)}\right) .
\end{aligned}
$$


The effective Lagrangian corresponding to this effective Hamiltonian is

$$
L_{\mathrm{eff}}=\int d \mathbf{x} \mathcal{L}_{\mathrm{Lan}}^{(+)}-H_{\mathrm{GF}+\mathrm{FP}}^{\prime},
$$

where $\mathcal{L}_{\text {Lan }}^{(+)}$is the Lagrangian density obtained by replacing $A_{0}, B, c$ and $\bar{c}$ by $A_{0(+)}, \tilde{B}_{(+)}, c_{(+)}$and $\bar{c}_{(+)}$, respectively. Thus, we have an effective theory with the zero modes removed from these fields and with an additional nonlocal interaction term $-H_{\mathrm{GF}+\mathrm{FP}}^{\prime}$ in the Lagrangian.

\section{EQUIVALENCE WITH THE FAIZAL-HIGUCHI PROPOSAL}

In this section we describe the proposal by Faizal and Higuchi [13] to solve the IR problem caused by the FP ghosts in de Sitter space and show that their proposal is equivalent to the use of the effective Lagrangian (107). They start from the FP-ghost propagator for a Lagrangian density with a small mass term $\sqrt{|g|} i M^{2} \bar{c} c$,

$$
T\left\langle 0\left|c^{a}(x) \bar{c}^{b}\left(x^{\prime}\right)\right| 0\right\rangle=i \delta^{a b} D_{M}\left(x, x^{\prime}\right) .
$$

Here, the state $|0\rangle$ is the perturbative Bunch-Davies vacuum state and where $D_{M}\left(x, x^{\prime}\right)$ is the propagator of the minimally coupled massive scalar field with mass $M$. This propagator can be given in terms of the variable $z=\cos ^{2}\left(H \mu\left(x, x^{\prime}\right) / 2\right)$, where $\mu\left(x, x^{\prime}\right)$ is the geodesic distance between the two points $x$ and $x^{\prime}$ when they are spacelike separated. (The function $\mu\left(x, x^{\prime}\right)$ can be analytically continued to the cases where $x$ and $x^{\prime}$ are not connected by a spacelike geodesic.) It is given by 35.

$$
D_{M}\left(x, x^{\prime}\right)=\frac{H^{n-2} \Gamma\left(b_{0+}\right) \Gamma\left(b_{0-}\right)}{(4 \pi)^{n / 2} \Gamma\left(\frac{n}{2}\right)} F\left(b_{0+}, b_{0-} ; \frac{n}{2} ; z\right),
$$

where $b_{0 \pm}$ are obtained by letting $\ell=0$ in Eq. (14). (Faizal and Higuchi work only on four-dimensional de Sitter space, but here we generalize their proposal to $n$ dimensions with $n \geq 2$.) For small $M$ we have

$$
D_{M}\left(x, x^{\prime}\right)=\frac{1}{2 c_{0} M^{2}}+O(1),
$$

where the constant $c_{0}$ is given by Eq. (18).

Now, the interaction term involving the FP ghosts in the Lagrangian density (54) is $-i \sqrt{|g|} \nabla^{\mu} \bar{c} \cdot\left(A_{\mu} \times c\right)$, in which the FP antighost is differentiated. This means that, if we use the propagator $\sqrt{109}$ in perturbation theory and then take the massless limit, the divergent constant term of the propagator does not contribute to the amplitude. With this observation, Faizal and Higuchi proposed to use the effective propagator obtained by subtracting the infinite constant from $D_{M}\left(x, x^{\prime}\right)$ :

$$
\begin{aligned}
& T\left\langle 0\left|c^{a}(x) \bar{c}^{b}\left(x^{\prime}\right)\right| 0\right\rangle_{\mathrm{eff}} \\
& =i \delta^{a b} \lim _{M \rightarrow 0^{+}}\left[D_{M}\left(x, x^{\prime}\right)-\frac{1}{2 c_{0} M^{2}}\right] .
\end{aligned}
$$

Note here that this propagator is de Sitter-invariant and IR finite.

We next describe this procedure of subtracting the IRdivergent constant in terms of mode expansion of the propagator. We discuss it in any spacetime with the metric of the form 71 with $N=1$. To start with we write down the Feynman propagator for the FP ghosts with $M \neq 0$ in terms of mode functions. One first chooses a complete set of solutions, $\varphi_{(n)}(t, \mathbf{x})$ (positive-frequency solutions) and $\varphi_{(n)}^{*}(t, \mathbf{x})$ (negative-frequency solutions), to the free-field equation $\left(\nabla_{\mu} \nabla^{\mu}+M^{2}\right) \varphi_{(n)}(t, \mathbf{x})=0$ such that

$$
\begin{aligned}
& i \int_{\Sigma} d \Sigma_{\mu}\left[\varphi_{(n)}^{*} \nabla^{\mu} \varphi_{(m)}-\varphi_{(m)} \nabla^{\mu} \varphi_{(n)}^{*}\right]=\delta_{m n} \\
& i \int_{\Sigma} d \Sigma_{\mu}\left[\varphi_{(n)} \nabla^{\mu} \varphi_{(m)}-\varphi_{(m)} \nabla^{\mu} \varphi_{(n)}\right]=0
\end{aligned}
$$

Then the free FP-ghost field operators (with small mass) can be expanded as

$$
\begin{aligned}
& c^{a}(t, \mathbf{x})=\sum_{n}\left[\alpha_{n}^{a} \varphi_{(n)}(t, \mathbf{x})+\alpha_{n}^{a \dagger} \varphi_{(n)}^{*}(t, \mathbf{x})\right] \\
& \bar{c}^{a}(t, \mathbf{x})=\sum_{n}\left[\bar{\alpha}_{n}^{a} \varphi_{(n)}(t, \mathbf{x})+\bar{\alpha}_{n}^{a \dagger} \varphi_{(n)}^{*}(t, \mathbf{x})\right]
\end{aligned}
$$

The equal-time canonical anticommutation relations are

$$
\begin{aligned}
\left\{\bar{c}^{a}(t, \mathbf{x}), \dot{c}^{b}\left(t, \mathbf{x}^{\prime}\right)\right\} & =-\left\{\dot{\bar{c}}^{a}(t, \mathbf{x}), c^{b}\left(t, \mathbf{x}^{\prime}\right)\right\} \\
& =\frac{\delta^{a b}}{\sqrt{\gamma(t, \mathbf{x})}} \delta\left(\mathbf{x}, \mathbf{x}^{\prime}\right),
\end{aligned}
$$

with all other equal-time anticommutators of the FP ghosts and their time derivatives vanishing. (Recall that we are assuming $N=1$.) These lead to

$$
\left\{\alpha_{n}^{a}, \bar{\alpha}_{m}^{b \dagger}\right\}=-\left\{\alpha_{n}^{a \dagger}, \bar{\alpha}_{m}^{b}\right\}=i \delta^{a b} \delta_{m n},
$$

with all other anticommutators among $\alpha_{n}^{a}, \bar{\alpha}_{m}^{a}$ and their Hermitian conjugates vanishing. The perturbative vacuum state $|0\rangle$ is annihilated by $\alpha_{n}^{a}$ and $\bar{\alpha}_{m}^{a}$. The Feynman propagator is then

$$
\begin{aligned}
& T\left\langle 0\left|c^{a}(t, \mathbf{x}) \bar{c}^{b}\left(t^{\prime}, \mathbf{x}^{\prime}\right)\right| 0\right\rangle \\
& =i \delta^{a b}\left[\theta\left(t-t^{\prime}\right) \sum_{n} \varphi_{(n)}(t, \mathbf{x}) \varphi_{(n)}^{*}\left(t^{\prime}, \mathbf{x}^{\prime}\right)\right. \\
& \left.\quad+\theta\left(t^{\prime}-t\right) \sum_{n} \varphi_{(n)}\left(t^{\prime}, \mathbf{x}^{\prime}\right) \varphi_{(n)}^{*}(t, \mathbf{x})\right]
\end{aligned}
$$

where $\theta\left(t-t^{\prime}\right)$ is the Heaviside step function.

The Klein-Gordon equation for the zero mode reads

$$
\frac{1}{V(t)} \frac{d}{d t}\left[V(t) \frac{d \varphi_{(0)}}{d t}\right]+M^{2} \varphi_{(0)}=0,
$$

where $V(t)$ is given by Eq. 83 with $N=1$. Two independent solutions with $M=0$ are 1 and $f(t)$, which 
is defined by Eq. 19]. Hence, for small $M$ the solution $\varphi_{(0)}$ can be given as

$$
\begin{aligned}
\varphi_{(0)}(t) \approx & \frac{1}{2 C(M)}\left\{1-M^{2}\left[g(t)+c_{3}\right]\right\} \\
& -i B(M)\left[f(t)+O\left(M^{2}\right)\right],
\end{aligned}
$$

where the function $g(t)$ is given by Eq. 20). We choose $C(M)$ to be real and positive, but $B(M)$ may be complex. In the $M \rightarrow 0^{+}$limit the $O\left(M^{2}\right)$ contribution in the last term can be neglected relative to the term proportional to $g(t)+c_{3}$ as long as $B(M) C(M) \rightarrow 0$ as $M \rightarrow 0^{+}$, which we assume. This is the case for the normalized positive-frequency zero modes in de Sitter space, where $B(M)$ and $C(M)$ are both of order $M$ (see Sec. II), and on the static flat torus, where they are of order $M^{1 / 2}$ (see Appendix E.

The normalization condition 112 implies

$$
\lim _{M \rightarrow 0^{+}} \frac{\operatorname{Re}[B(M)]}{C(M)}=1 .
$$

We assume that $B(M)$ and $C(M)$ are of order $M^{\alpha}$ with $0<\alpha \leq 1$. (For the de Sitter case we have $\alpha=1$ whereas for the static flat torus we have $\alpha=1 / 2$ as we pointed out above.) Thus, $B(M), C(M)$ and $M^{2} / C(M)$ tend to zero as $M \rightarrow 0^{+}$. However, the limit of $M / C(M)$ as $M \rightarrow 0^{+}$is nonzero if $\alpha=1$.

The zero-mode sector of the Feynman propagator for the FP ghosts is IR divergent, and its divergent term reads

$$
\begin{aligned}
D^{(0) a b}\left(t, t^{\prime}\right) & =i \delta^{a b}\left[\theta\left(t-t^{\prime}\right) \varphi_{(0)}(t) \varphi_{(0)}^{*}\left(t^{\prime}\right)+\left(t \leftrightarrow t^{\prime}\right)\right] \\
& =\frac{i \delta^{a b}}{4 C^{2}(M)}+O(1) .
\end{aligned}
$$

The subtraction of the IR-divergent term from the FPghost propagator changes the zero-mode contribution as follows:

$$
\begin{aligned}
D_{\mathrm{eff}}^{(0) a b}\left(t, t^{\prime}\right):= & \lim _{M \rightarrow 0^{+}}\left[D^{(0) a b}\left(t, t^{\prime}\right)-\frac{i \delta^{a b}}{4 C^{2}(M)}\right] \\
= & \delta^{a b}\left\{-i \beta_{0}^{2}\left[g(t)+g\left(t^{\prime}\right)\right]+\frac{i \beta_{1}}{2}\left[f(t)+f\left(t^{\prime}\right)\right]\right. \\
& \left.+\frac{1}{2}\left[\theta\left(t-t^{\prime}\right)-\theta\left(t^{\prime}-t\right)\right]\left[f(t)-f\left(t^{\prime}\right)\right]\right\},
\end{aligned}
$$

where the constants $\beta_{0}, \beta_{1}$ are given by

$$
\begin{aligned}
& \beta_{0}:=\lim _{M \rightarrow 0^{+}} \frac{M}{C(M)}, \\
& \beta_{1}:=\lim _{M \rightarrow 0^{+}} \frac{\operatorname{Im}[B(M)]}{C(M)} .
\end{aligned}
$$

We have used Eq. 121) in the last term of Eq. 123. If $C(M)=O\left(M^{\alpha}\right)$ with $\alpha<1$, then $\beta_{0}=0$. For the de Sitter case we have $\alpha=1$ and $\beta_{0}^{2}=2 / c_{0}$ [see Eq. [17)].

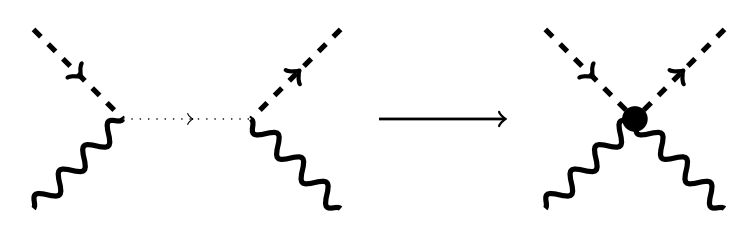

FIG. 1. The wavy, dashed and dotted lines represent the gauge field, the nonzero-mode part of the FP-ghost propagator and its zero-mode part, respectively. The zero-mode contribution to the FP-ghost propagator is integrated out in Eq. 128 by using Eq. 129.

Now, the interaction term in the Lagrangian density involving the FP ghosts can be written as

$$
-i \sqrt{|g|} q \nabla_{\mu} \bar{c} \cdot\left(A^{\mu} \times c\right) \text { or } i \sqrt{|g|} q \nabla_{\mu} c \cdot\left(A^{\mu} \times \bar{c}\right) .
$$

Here, we have assumed that one can freely integrate by parts at vertices with no boundary terms. This might appear problematic in de Sitter space, where the boundaries at the past and future grow exponentially. However, it turns out that one can construct an in-in formalism [36, 37] for which the boundary terms vanish upon integration by parts in this spacetime [38]. (For early use of the in-in formalism in curved spacetime, see, e.g. Refs. 39 42.) Therefore, we proceed under the assumption that integration by parts does not generate nonzero surface terms at the vertex where the FP ghosts interact with the gauge field. (We emphasize, however, that this assumption is necessary only for the equivalence of our method and the Faizal-Higuchi proposal. Our method itself is valid even if integration by parts generates nonzero surface terms.) Note also that we have set $\nabla_{\mu} A^{\mu}=0$. This is valid because in the Landau gauge the Feynman propagator for the gauge field is divergence-free.

The interaction term involving the zero modes of the FP ghosts can then be written as

$$
\begin{aligned}
& I_{c}=-i \sqrt{|g|} q \partial_{0} \bar{c}_{(0)} \cdot\left(\varpi_{\tilde{B}(+)} \times c_{(+)}\right), \\
& I_{\bar{c}}=-i \sqrt{|g|} q\left(\varpi_{\tilde{B}(+)} \times \bar{c}_{(+)}\right) \cdot \partial_{0} c_{(0)} .
\end{aligned}
$$

We have dropped the terms that become zero upon integration over the spacetime, or more precisely, over the space and the chosen time path in the appropriate in-in formalism. For example, terms of the form $X_{(0)} Y_{(0)} Z_{(+)}$ vanish upon integration over the space. (In general we need to subtract the zero-mode contribution to $A_{0}$ from the propagator of the gauge field to impose the condition $A_{0(0)}|0\rangle=-\varpi_{\tilde{B}(0)}|0\rangle=0$ on the perturbative vacuum $|0\rangle$. Interestingly, for the de Sitter case this condition follows automatically from de Sitter invariance as shown in Appendix $\mathrm{F}$.)

Since the zero-mode contribution to the fields $c$ and $\bar{c}$ appears only once in each of the interaction terms (126) and (127), one can integrate out the zero-mode contribution in perturbation theory as shown in Fig. 1. This integration introduces the following extra term in the La- 
grangian:

$$
\begin{aligned}
L^{\operatorname{extra}}(t)= & -i \int d \mathbf{x} \sqrt{|\gamma(t, \mathbf{x})|} \int d t^{\prime} d \mathbf{x}^{\prime} \sqrt{\left|\gamma\left(t^{\prime}, \mathbf{x}\right)\right|} \\
& \times\left[\varpi_{\tilde{B}(+)}(t, \mathbf{x}) \times \bar{c}_{(+)}(t, \mathbf{x})\right]^{a} \frac{\partial^{2}}{\partial t \partial t^{\prime}} D_{\mathrm{eff}}^{(0) a b}\left(t, t^{\prime}\right) \\
& \times\left[\varpi_{\tilde{B}(+)}\left(t^{\prime}, \mathbf{x}^{\prime}\right) \times c_{(+)}\left(t^{\prime}, \mathbf{x}^{\prime}\right)\right]^{b}
\end{aligned}
$$

Now we find from Eq. 123

$$
\frac{\partial^{2}}{\partial t \partial t^{\prime}} D_{\mathrm{eff}}^{(0) a b}\left(t, t^{\prime}\right)=-\delta^{a b} \frac{1}{V(t)} \delta\left(t-t^{\prime}\right) .
$$

By substituting this expression into Eq. 128 we obtain $L^{\text {extra }}=-H_{\mathrm{GF}+\mathrm{FP}}^{\prime}$, where $H_{\mathrm{GF}+\mathrm{FP}}^{\prime}$ is given by Eq. 104 . Thus, Lagrangian perturbation theory with the effective FP-ghost propagator 111 proposed in Ref. [13] agrees with perturbation theory using the effective Lagrangian (107), which has been derived by imposing the conditions (66).

\section{SUMMARY AND DISCUSSION}

In this paper we showed that the space-independent modes of the Faddeev-Popov ghosts can be removed from the covariantly quantized Yang-Mills theory in spacetime with compact spatial sections in the Landau gauge by requiring that the Noether charges corresponding to the conserved currents $A^{\mu}, D^{\mu} c$ and $D^{\mu} \bar{c}$ annihilate the physical states. There will be no IR divergences or de Sitter symmetry breaking from the ghost sector if these conditions are applied to Yang-Mills theory in global de Sitter space. We also showed that the compatibility of these conditions with the invariance of the physical states under the BRST transformation leads to the requirement that the physical states be invariant under the global transformations corresponding to the gauge group. Then we showed that the theory resulting from removing the FP-ghost zero modes in this manner is equivalent to using the effective FP-ghost propagator with the constant IRdivergent term subtracted. This propagator is de Sitterinvariant, which confirms that our method of removing the zero modes is de Sitter-invariant when applied to de Sitter space.

The IR issues in perturbative gravity in de Sitter space have been debated since the physical ${ }^{1}$ graviton two-point function in the conformally flat, or Poincaré, patch was found to be IR divergent 43. However, it has been demonstrated that the IR divergences in this two-point function are gauge artifact in the sense that they can be expressed in a pure-gauge form [44 46] and that the mode functions can be chosen to make the two-point

\footnotetext{
1 Here, the two-point function is called "physical" in the sense that the gauge degrees of freedom are completely fixed.
}

function IR finite 47]. IR-finite physical graviton twopoint functions have also been constructed in other coordinate patches of de Sitter space [48 50]. The IR-finite graviton propagators in covariant gauges have also been derived 51 53. It has been shown that these propagators can be constructed by the mode-sum method in the global patch of de Sitter space provided that the Euclidean massive tensor-field propagator is obtained using this method [54]. (The mode summation in the Poincaré patch, on the other hand, appears to lead to an IR-divergent graviton propagator even in the covariant gauge [55, 56] though the IR-divergent terms are of puregauge form.) Moreover, the gauge-invariant two-point function as defined in Ref. [57] is known to be equivalent to that of the linearized Weyl tensor [58, which is known to be IR finite and de Sitter-invariant in the Bunch-Davies-like vacuum state 59 61]. Thus, in fact there are no IR problems in noninteracting linearized gravity. However, it is not clear whether or not the IR divergences of the graviton propagator in the Poincaré patch will lead to physical effects, such as the breakdown of de Sitter symmetry, in perturbative gravity. Some authors claim they will (see, e.g. Ref. 62]) but other authors suggest they will not (see, e.g. Ref. 63]).

We note in this context that the de Sitter-invariant FP-ghost propagator in the covariant gauge is also IR divergent. Hence, one might expect that the IR effect in the FP-ghost sector would lead to breakdown of de Sitter symmetry. However, the interaction terms involving the FP ghosts are such that the IR-divergent part of the propagator does not contribute if one regularizes the IR divergences by a small mass term as in the Yang-Mills case treated in this paper. Because of this fact it was proposed in Ref. [13] that one should simply subtract the IR-divergent contribution from the FP-ghost propagator to obtain an effective de Sitter invariant propagator, as in the Yang-Mills case.

Now, it is not difficult to see that there are conserved Noether charges analogous to those found in Sec. III in covariantly quantized perturbative gravity in the Landau gauge. Thus, we expect that the modes of the FP ghosts responsible for IR divergences will be removed if the physical states are required to be annihilated by these conserved charges. If this is the case, it will be interesting to find out whether or not the effective theory thus obtained is equivalent to the proposal of Ref. [13] in perturbative gravity as in Yang-Mills theory. This question is currently under investigation.

\section{ACKNOWLEDGMENTS}

We thank Mir Faizal, Chris Fewster, Markus Fröb and Albert Roura for useful discussions. The work of J. G. was supported by the Engineering and Physical Sciences Research Council (EPSRC). 


\section{Appendix A: The zero mode of the minimally coupled scalar field in de Sitter space}

Let us first examine the small $M$ limit of the hypergeometric function in Eq. (13) with $\ell=0$. We use the formula 9.136.1 in Ref. 64,

$$
\begin{aligned}
& F\left(2 \alpha, 2 \beta ; \alpha+\beta+\frac{1}{2} ; \frac{1-\sqrt{z}}{2}\right) \\
& =A F\left(\alpha, \beta ; \frac{1}{2} ; z\right)+B \sqrt{z} F\left(\alpha+\frac{1}{2}, \beta+\frac{1}{2} ; \frac{3}{2} ; z\right),
\end{aligned}
$$

where

$$
\begin{aligned}
& A=\frac{\Gamma\left(\alpha+\beta+\frac{1}{2}\right) \sqrt{\pi}}{\Gamma\left(\alpha+\frac{1}{2}\right) \Gamma\left(\beta+\frac{1}{2}\right)}, \\
& B=-\frac{\Gamma\left(\alpha+\beta+\frac{1}{2}\right) 2 \sqrt{\pi}}{\Gamma(\alpha) \Gamma(\beta)},
\end{aligned}
$$

with $z=-\sinh ^{2} t$ and $\sqrt{z}=i \sinh t$. We find

$$
\begin{aligned}
& F\left(b_{0+}, b_{0-}, \ell+\frac{n}{2} ; \frac{1-i \sinh H t}{2}\right) \\
& =A F\left(\frac{b_{0+}}{2}, \frac{b_{0-}}{2} ; \frac{1}{2} ;-\sinh ^{2} H t\right) \\
& +i B \sinh H t F\left(\frac{n}{2}, \frac{1}{2} ; \frac{3}{2} ;-\sinh ^{2} H t\right),
\end{aligned}
$$

where

$$
\begin{aligned}
& A \approx 1+\left[\psi\left(\frac{n}{2}\right)-\psi\left(\frac{1}{2}\right)\right] \frac{M^{2}}{2(n-1) H^{2}}, \\
& B \approx-\frac{\Gamma\left(\frac{n}{2}\right) \sqrt{\pi} M^{2}}{2 \Gamma\left(\frac{n+1}{2}\right) H^{2}} .
\end{aligned}
$$

Recall that $\psi(x):=\Gamma^{\prime}(x) / \Gamma(x)$ and

$$
\begin{aligned}
b_{0 \pm} & =\frac{n-1}{2} \pm \sqrt{\left(\frac{n-1}{2}\right)^{2}-\frac{M^{2}}{H^{2}}} \\
& \approx \frac{n-1}{2} \pm\left(\frac{n-1}{2}-\frac{M^{2}}{(n-1) H^{2}}\right) .
\end{aligned}
$$

One can readily show that

$$
\begin{aligned}
& \frac{1}{H} \frac{d}{d t}\left[\sinh H t F\left(\frac{n}{2}, \frac{1}{2} ; \frac{3}{2} ;-\sinh ^{2} H t\right)\right], \\
& =(\cosh H t)^{-(n-1)}
\end{aligned}
$$

using the series expression of the hypergeometric function. Thus,

$$
\begin{aligned}
& \sinh H t F\left(\frac{n}{2}, \frac{1}{2} ; \frac{3}{2} ;-\sinh ^{2} H t\right) \\
& =H \int_{0}^{t} \frac{d t^{\prime}}{(\cosh H t)^{n-1}} .
\end{aligned}
$$

We find in a similar manner, noting that $\left.b_{0-}\right|_{M=0}=0$ and using Eq. 12p,

$$
\begin{aligned}
& F\left(\frac{b_{0+}}{2}, \frac{b_{0-}}{2} ; \frac{1}{2} ;-\sinh ^{2} H t\right) \\
& \approx 1-M^{2} \int_{0}^{t} d t^{\prime}\left(\cosh H t^{\prime}\right)^{-(n-1)} \\
& \quad \times \int_{0}^{t^{\prime}} d t^{\prime \prime}\left(\cosh H t^{\prime \prime}\right)^{n-1} .
\end{aligned}
$$

Then, substituting Eqs. (A5), (A6), (A9) and (A10) into Eq. A4, we find

$$
\begin{aligned}
& F\left(b_{0+}, b_{0-} ; \ell+\frac{n}{2} ; \frac{1-i \sinh H t}{2}\right) \\
& \approx 1-M^{2}\left\{g(t)-\left[\psi\left(\frac{n}{2}\right)-\psi\left(\frac{1}{2}\right)\right] \frac{1}{2(n-1) H^{2}}\right\} \\
& -i c_{0} M^{2} f(t),
\end{aligned}
$$

where the functions $f(t)$ and $g(t)$ are defined by Eqs. (19) and (20), respectively, and the constant $c_{0}$ is given by Eq. (18).

The normalized zero mode $F_{0}(t)=f_{0}(t) Y_{00}(\boldsymbol{\theta})$, where $f_{0}(t)$ can be found by letting $\ell=0$ in Eq. (13), is obtained by multiplying the hypergeometric function in Eq. A11) by

$$
K_{0}=\frac{H^{\frac{n-2}{2}} N_{0}}{2^{\frac{n-2}{2}} \Gamma\left(\frac{n}{2}\right)} Y_{00}(\boldsymbol{\theta}) .
$$

Recalling that $Y_{00}(\boldsymbol{\theta})=V_{S^{n-1}}^{-1 / 2}$, where $V_{S^{n-1}}$ is the volume of the unit $S^{n-1}$ given by Eq. 222, and using the expression of $N_{\ell}$ with $\ell=0$ in Eq. (7) (choosing the convention $N_{0}>0$ ), we find

$$
K_{0}=\left(\frac{H^{n-2} \Gamma\left(\frac{n-1}{2}+\lambda\right) \Gamma\left(\frac{n-1}{2}-\lambda\right)}{2^{n} \pi^{n / 2} \Gamma\left(\frac{n}{2}\right)}\right)^{1 / 2}
$$

For small $M$ we obtain

$$
K_{0} \approx \frac{1}{\sqrt{2 c_{0}} M}\left\{1-[\psi(n-1)-\psi(1)] \frac{M^{2}}{2(n-1) H^{2}}\right\},
$$

where we have used the doubling formula:

$$
\Gamma(2 x)=\frac{2^{2 x-1}}{\sqrt{\pi}} \Gamma(x) \Gamma\left(x+\frac{1}{2}\right) .
$$

Multiplying Eq. (A11) by $K_{0}$ in Eq. A14, we indeed obtain Eq. 17] with

$$
c_{1}=\frac{1}{2(n-1) H^{2}}\left[\psi(n-1)-\psi\left(\frac{n}{2}\right)-\psi(1)+\psi\left(\frac{1}{2}\right)\right] .
$$

Next we examine the action of the boost Killing vector $L_{X}$ defined by Eq. 30 on the mode functions $F_{\ell m \tilde{\sigma}}(t, \boldsymbol{\theta})$ 
and derive Eq. (31), following Ref. [15. We first note that the Killing vector $L_{X}$ can be written as

$$
L_{X}=T^{(+)} S^{(+)}+T^{(-)} S^{(-)},
$$

where

$$
\begin{aligned}
& T^{(+)}=\frac{1}{H} \frac{\partial}{\partial t}-\ell \tanh H t, \\
& T^{(-)}=\frac{1}{H} \frac{\partial}{\partial t}+(\ell+n-2) \tanh H t,
\end{aligned}
$$

and

$$
\begin{aligned}
& S^{(+)}=\frac{1}{2 \ell+n-2}\left[\sin \chi \frac{\partial}{\partial \chi}+(\ell+n-2) \cos \chi\right], \\
& S^{(-)}=-\frac{1}{2 \ell+n-2}\left[\sin \chi \frac{\partial}{\partial \chi}-\ell \cos \chi\right] .
\end{aligned}
$$

By using the raising and lowering operators for the associated Legendre functions found in 8.733.1 of Ref. [64, we obtain

$$
\begin{aligned}
& T^{(+)} f_{\ell}(t)=-i\left[\ell(\ell+n-1)+\frac{M^{2}}{H^{2}}\right]^{1 / 2} f_{\ell+1}(t), \quad(\mathrm{A} 22) \\
& T^{(-)} f_{\ell}(t)=-i\left[(\ell-1)(\ell+n-2)+\frac{M^{2}}{H^{2}}\right]^{1 / 2} f_{\ell-1}(t),
\end{aligned}
$$

where Eq. A23 is for $\ell \geq 1$, and

$$
\begin{aligned}
& S^{(+)} Y_{\ell m \tilde{\sigma}}=\left[\frac{(\ell-m+1)(\ell+m+n-2)}{(2 \ell+n)(2 \ell+n-2)}\right]^{1 / 2} Y_{(\ell+1) m \tilde{\sigma}}, \\
& S^{(-)} Y_{\ell m \tilde{\sigma}}=\left[\frac{(\ell-m)(\ell+m+n-3)}{(2 \ell+n-2)(2 \ell+n-4)}\right]^{1 / 2} Y_{(\ell-1) m \tilde{\sigma}} .
\end{aligned}
$$

By using these formulas and the decomposition A17 we find Eq. 31.

\section{Appendix B: The effective Hamiltonian in general spacetime}

In this appendix we present the effective Hamiltonian in spacetime with the more general line element of the Arnowitt-Deser-Misner metric 65, given by

$$
d s^{2}=N^{2} d t^{2}-\gamma_{i j}\left(d x^{i}+N^{i} d t\right)\left(d x^{j}+N^{j} d t\right),
$$

where the lapse function $N$, the shift vector $N^{i}$ and the metric $\gamma_{i j}$ on space depend on $t$ and $\mathbf{x}$ in general. The standard Hamiltonian density $\mathcal{H}_{\text {st }}$ is given as

$$
\begin{aligned}
\frac{1}{\sqrt{|g|}} \mathcal{H}_{\mathrm{st}}= & \frac{1}{\sqrt{|g|}} \mathcal{H}_{\text {class }}+A^{i} \cdot\left(\nabla_{i} B-i q \nabla_{i} \bar{c} \times c\right) \\
& -\frac{i}{N^{2}} \nabla_{0} \bar{c} \cdot \nabla_{0} c \\
& -i\left(\gamma^{i j}-\frac{N^{i} N^{j}}{N^{2}}\right) \nabla_{i} \bar{c} \cdot \nabla_{j} c,
\end{aligned}
$$

where the classical Hamiltonian density $\mathcal{H}_{\text {class }}$ is given by the following formula:

$$
\frac{1}{\sqrt{|g|}} \mathcal{H}_{\text {class }}=-\frac{1}{2} F_{0 i} \cdot F^{0 i}+\frac{1}{4} F_{i j} \cdot F^{i j}+\frac{1}{N^{2}} D_{i} A_{0} \cdot \pi^{i}
$$

with

$$
F^{0 i}=-N^{-2} \pi^{i}
$$

We again define $\tilde{B}:=B-i q \bar{c} \times c_{(0)}$, where $c_{(0)}$ is defined by Eq. 82 with $V(t)$ given by Eq. 83 . We again define the conjugate momentum densities of $\tilde{B}, c$ and $\bar{c}$ multiplied by $N / \sqrt{\gamma}$ as $\varpi_{\tilde{B}}, \varpi_{c}$ and $\varpi_{\bar{c}}$. They are given by

$$
\begin{aligned}
& \varpi_{\tilde{B}}=-N^{2} A^{0}, \\
& \varpi_{c}=i N^{2} \nabla^{0} \bar{c}-\frac{i q}{V(t)} \int d \mathbf{x} \frac{\sqrt{\gamma}}{N}\left(\varpi_{\tilde{B}} \times \bar{c}\right), \\
& \varpi_{\bar{c}}=-i N^{2}\left(\nabla^{0} c+q A^{0} \times c_{(+)}\right) .
\end{aligned}
$$

The zero modes $\varpi_{\tilde{B}(0)}, \varpi_{c(0)}$ and $\varpi_{\bar{c}(0)}$ defined as in Sec. III are related to the conserved charges $Q_{A}, Q_{D c}$ and $Q_{D \bar{c}}$ as in Eqs. (98)-(100). Therefore, we can require the conditions $\varpi_{\tilde{B}(0)} \mid$ phys $\rangle=\varpi_{c(0)} \mid$ phys $\rangle=\varpi_{\bar{c}(0)} \mid$ phys $\rangle=0$ for the physical states $\mid$ phys $\rangle$.

We have $\varpi_{\tilde{B}}=\varpi_{B}$ but the conjugate momentum densities for $c$ and $\bar{c}$ differ from the ones before the variable change by the following quantities:

$$
\begin{aligned}
& \Delta \varpi_{c}=-\frac{i q}{V(t)} \int d \mathbf{x} \frac{\sqrt{\gamma}}{N}\left(\varpi_{\tilde{B}} \times \bar{c}\right), \\
& \Delta \varpi_{\bar{c}}=-i q \varpi_{\tilde{B}} \times c_{(0)} .
\end{aligned}
$$

The Hamiltonian after the change of variables $\tilde{B}=$ $B-i q \bar{c} \times c_{(0)}$ is different from the standard Hamiltonian even when it is expressed in terms of the fields and their time derivatives (rather than their canonical conjugate momenta) because this change of variables explicitly depends on $t$. We note that this is not the case with the metric chosen in Sec. IV because the definition of $c_{(0)}$ there does not depend on $t$ explicitly. The difference $\Delta \mathcal{H}$ between the Hamiltonian density $\mathcal{H}$ after the variable change and the standard Hamiltonian density $\mathcal{H}_{\text {st }}$ is given as

$$
\begin{aligned}
\frac{1}{\sqrt{|g|}} \Delta \mathcal{H}= & \frac{1}{\sqrt{|g|}}\left(\mathcal{H}-\mathcal{H}_{\mathrm{st}}\right) \\
= & N^{-2} \frac{\partial}{\partial t}\left(-i q \bar{c} \times c_{(0)}\right) \cdot \varpi_{\tilde{B}} \\
& +N^{-2}\left(-\Delta \varpi_{c} \cdot \dot{c}+\dot{\bar{c}} \cdot \Delta \varpi_{\bar{c}}\right) .
\end{aligned}
$$


Then

$$
\begin{aligned}
\Delta H:= & \int d \mathbf{x} \Delta \mathcal{H} \\
= & -i q \int d \mathbf{x} \frac{\sqrt{\gamma}}{N}\left(\varpi_{\tilde{B}} \times \bar{c}\right) \\
& \cdot\left[\frac{\partial}{\partial t}\left(\int d \mathbf{y} \frac{\sqrt{\gamma}}{V(t) N} c(t, \mathbf{y})\right)-\int d \mathbf{y} \frac{\sqrt{\gamma}}{V(t) N} \frac{\partial}{\partial t} c(t, \mathbf{y})\right] \\
= & -i q \int d \mathbf{x} \frac{\sqrt{\gamma}}{N}\left(\varpi_{\tilde{B}} \times \bar{c}\right) \cdot \int d \mathbf{y} \frac{\partial}{\partial t}\left[\frac{\sqrt{\gamma}}{V(t) N}\right] c_{(+)}(t, \mathbf{y}) .
\end{aligned}
$$

It is clear that $\Delta H=0$ for the metric chosen in Sec. IV because $\sqrt{\gamma} /(V N)$ is time independent there. We have replaced $c(t, \mathbf{y})$ by $c_{(+)}(t, \mathbf{y})$ because

$$
\int d \mathbf{y} \frac{\partial}{\partial t}\left[\frac{\sqrt{\gamma}}{V(t) N}\right]=0 .
$$

The effective Hamiltonian for the physical states $\mid$ phys $\rangle$ satisfying $\varpi_{\tilde{B}(0)} \mid$ phys $\rangle=\varpi_{c(0)} \mid$ phys $\rangle=\varpi_{\bar{c}(0)} \mid$ phys $\rangle=0$ can be obtained by solving Eqs. (B5)-(B7) for $A^{0}, \nabla_{0} \bar{c}$ and $\nabla_{0} c$, and substituting the results and $B=\tilde{B}+i q \bar{c} \times$ $c_{(0)}$ in Eq. $\mathrm{B} 2$ and adding $\Delta H$ given by Eq. (B11). The result can be given in the following form:

$$
H_{\text {eff }}=\int d \mathbf{x} \mathcal{H}_{\text {conv }}-\frac{i q}{V(t)} \bar{F} \cdot \widetilde{F} .
$$

Here, the "conventional" Hamiltonian density $\mathcal{H}_{\text {conv }}$ is given by

$$
\begin{aligned}
\mathcal{H}_{\text {conv }}= & \mathcal{H}_{\text {class }}+\sqrt{\gamma} N A^{i} \cdot\left(\nabla_{i} \tilde{B}-i q \nabla_{i} \bar{c} \times c\right) \\
& -\frac{\sqrt{\gamma}}{N} \varpi_{c} \cdot\left[i \varpi_{\bar{c}}+N^{i} \nabla_{i} c+q \varpi_{\tilde{B}} \times c\right] \\
& +\frac{\sqrt{\gamma}}{N} N^{i} \nabla_{i} \bar{c} \cdot\left(\varpi_{\bar{c}}-i q \varpi_{\tilde{B}} \times c\right) \\
& +\sqrt{\gamma} N \gamma^{i j} \nabla_{i} \bar{c} \cdot \nabla_{j} c,
\end{aligned}
$$

where the fields $c, \bar{c}, B$ and their (rescaled) conjugate momentum densities $\varpi_{c}, \varpi_{\bar{c}}$ and $\varpi_{\tilde{B}}$ are understood to be their nonzero-mode contributions, $c_{(+)}, \bar{c}_{(+)}, \tilde{B}_{(+)}$, $\varpi_{c(+)}, \varpi_{\bar{c}(+)}$ and $\varpi_{\tilde{B}(+)}$. The operators $\bar{F}$ and $\widetilde{F}$ are given by

$$
\begin{aligned}
\bar{F}:= & \int d \mathbf{x} \frac{\sqrt{\gamma}}{N}\left(\varpi_{B(+)} \times \bar{c}_{(+)}\right), \\
\widetilde{F}:= & \int d \mathbf{x} \frac{\sqrt{\gamma}}{N} \\
& \times\left[\frac{N}{\sqrt{\gamma}} \frac{\partial}{\partial t}\left(\frac{\sqrt{\gamma}}{N}\right) c_{(+)}+q \varpi_{\tilde{B}(+)} \times c_{(+)}+N^{i} \nabla_{i} c_{(+)}\right] .
\end{aligned}
$$

In principle one can use the Hamiltonian $H_{\text {eff }}$ here for perturbative calculations though it may not be very practical to do so.

\section{Appendix C: The Dirac bracket}

In this paper we identified $-\sqrt{|g|} A^{0}$ with the canonical momentum density for $B$ (and $\tilde{B}$ ), and did not regard it as an independent dynamical variable. In this appendix we show that this treatment agrees with the quantization using the Dirac bracket. (The variables $B$ and $\pi_{B}$ may be replaced by $\tilde{B}$ and $\pi_{\tilde{B}}$ below without any other changes.) We suppress the argument $t$ since all brackets are computed at an equal time.

We have two second-class constraints :

$$
\begin{aligned}
& C_{1}:=|g|^{-1 / 2} \pi_{B}+A^{0} \approx 0, \\
& C_{2}:=\pi_{A^{0}} \approx 0,
\end{aligned}
$$

where $\pi_{A^{0}}$ is the canonical conjugate momentum density for $A^{0}$. The Poisson bracket for these constraints is

$$
\left\{C_{I}(\mathbf{x}), C_{J}\left(\mathbf{x}^{\prime}\right)\right\}_{\mathrm{PB}}=\delta\left(\mathbf{x}, \mathbf{x}^{\prime}\right) \epsilon_{I J},
$$

where $\epsilon_{I J}$ is the antisymmetric $2 \times 2$ matrix with $\epsilon_{12}=1$. The inverse of this Poisson bracket is

$$
\Delta^{I J}\left(\mathbf{x}, \mathbf{x}^{\prime}\right)=-\delta\left(\mathbf{x}, \mathbf{x}^{\prime}\right) \epsilon^{I J},
$$

where $\epsilon^{I J}$ is identical with $\epsilon_{I J}$ as a matrix.

The Dirac bracket for two canonical variables $X(\mathbf{x})$ and $Y\left(\mathbf{x}^{\prime}\right)$ can be computed as

$\left\{X(\mathbf{x}), Y\left(\mathbf{x}^{\prime}\right)\right\}_{\mathrm{DB}}$

$=\left\{X(\mathbf{x}), Y\left(\mathbf{x}^{\prime}\right)\right\}_{\mathrm{PB}}$

$-\int d \mathbf{y} \int d \mathbf{z}\left\{X(\mathbf{x}), C_{I}(\mathbf{y})\right\}_{\mathrm{PB}} \Delta^{I J}(\mathbf{y}, \mathbf{z})\left\{C_{J}(\mathbf{z}), Y\left(\mathbf{x}^{\prime}\right)\right\}_{\mathrm{PB}}$

$=\left\{X(\mathbf{x}), Y\left(\mathbf{x}^{\prime}\right)\right\}_{\mathrm{PB}}$

$+\int d \mathbf{y}\left\{X(\mathbf{x}), C_{I}(\mathbf{y})\right\}_{\mathrm{PB}}\left\{C_{J}(\mathbf{y}), Y\left(\mathbf{x}^{\prime}\right)\right\}_{\mathrm{PB}} \epsilon^{I J}$.

Then, by construction $\left\{C_{I}(\mathbf{x}), X\left(\mathbf{x}^{\prime}\right)\right\}_{\mathrm{DB}}=0$ for all $C_{I}(\mathbf{x})$ and $X\left(\mathbf{x}^{\prime}\right)$. We also have

$$
\left\{B(\mathbf{x}), \pi_{B}\left(\mathbf{x}^{\prime}\right)\right\}_{\mathrm{DB}}=\left\{B(\mathbf{x}), \pi_{B}\left(\mathbf{x}^{\prime}\right)\right\}_{\mathrm{PB}}=\delta\left(\mathbf{x}, \mathbf{x}^{\prime}\right) .
$$

Hence, if we "promote" the Dirac bracket to commutation relations, we find that the operators $\pi_{A^{0}}$ and $\pi_{B}+\sqrt{|g|} A^{0}$ commute with all operators and that $B$ and $\pi_{B}$ satisfy the standard commutation relations. Hence, basing the canonical commutation relations on the Dirac bracket is equivalent to letting $\pi_{A^{0}}=0$ and $\pi_{B}=$ $-\sqrt{|g|} A^{0}$, as we did in this paper.

\section{Appendix D: Redefining the inner product}

As was pointed out in Sec. IV] if we represent the state $\mid$ phys $\rangle$ as a wave functional $\Psi[B(\mathbf{x}), c(\mathbf{x}), \bar{c}(\mathbf{x})]$, then the conditions $\varpi_{\tilde{B}(0)} \mid$ phys $\rangle=\varpi_{c(0)} \mid$ phys $\rangle=\varpi_{\bar{c}(0)} \mid$ phys $\rangle=0$ can be translated to the following equations:

$$
\frac{\partial}{\partial \tilde{B}_{(0)}} \Psi=0
$$


and

$$
\frac{\partial}{\partial c_{(0)}} \Psi=\frac{\partial}{\partial \bar{c}_{(0)}} \Psi=0 .
$$

Now the wave functional $\Psi$ is formally normalized by the condition

$$
\int[d \tilde{B}][d c][d \bar{c}]|\Psi[\tilde{B}, c, \bar{c}]|^{2}=1 .
$$

This functional integral contains the integrations over the variables $\tilde{B}_{(0)}, c_{(0)}$ and $\bar{c}_{(0)}$. Since the functional $\Psi$ is independent of these variables due to Eqs. (D1) and (D2), we have

$$
\begin{aligned}
\int d \tilde{B}_{(0)}|\Psi|^{2} & =\infty \\
\int d c_{(0)}|\Psi|^{2} & =\int d \bar{c}_{(0)}|\Psi|^{2}=0 .
\end{aligned}
$$

Equation (D5) follows from the rule that the integration with respect to a Grassmann variable is the same as the (left) differentiation [66, 67. Thus, the normalization integral (D3) will be (even more) ill-defined. However, this situation can readily be remedied by dropping the variables $\tilde{B}_{(0)}, c_{(0)}$ and $\bar{c}_{(0)}$ from the normalization integral.

In the rest of this appendix, we present a 2 -dimensional representation of the fermionic commutation relations $\theta^{2}=\pi_{\theta}^{2}=0,\left\{\pi_{\theta}, \theta\right\}=-i$, where $\theta^{\dagger}=\theta$. We expect from Eq. (D5) that a state annihilated by $\pi_{\theta}$ has zero norm. It is clear that the nilpotency of the Hermitian operator $\theta$ requires an indefinite pseudoinner product because the square of a nonzero Hermitian operator cannot vanish in a Hilbert space with a positive-definite inner product. We introduce a pseudoinner product on the 2-dimensional vector space $\operatorname{Span}\{|0\rangle,|1\rangle\}$ by $\langle 0 \mid 0\rangle=\langle 1 \mid 1\rangle=0,\langle 0 \mid 1\rangle=$ $\langle 1 \mid 0\rangle=1$. We let $\theta|0\rangle=|1\rangle$ and $\theta|1\rangle=0$. We find $\theta^{2}=0$ and that $\theta$ is Hermitian: $\langle 0|\theta| 1\rangle=\langle 1|\theta| 0\rangle=0$, $\langle 0|\theta| 0\rangle=1$ and $\langle 1|\theta| 1\rangle=0$. The operator $\pi_{\theta}$ can be represented as $\pi_{\theta}|0\rangle=0, \pi_{\theta}|1\rangle=-i|0\rangle$. Then we find $\pi_{\theta}^{2}=0$ and $\left\{\pi_{\theta}, \theta\right\}=-i$. (Note that $\pi_{\theta}$ is antiHermitian.) The state $|0\rangle$ satisfying $\pi_{\theta}|0\rangle=0$ indeed has zero norm: $\langle 0 \mid 0\rangle=0$.

\section{Appendix E: The flat-torus case}

In this appendix we discuss some results of this paper in the special case where the spacetime corresponds to a static flat torus. We let the torus have volume $V$. The normalized positive-frequency zero mode with small mass $M$ is

$$
\begin{aligned}
\varphi_{(0)}(t) & =\frac{1}{\sqrt{2 V M}} e^{-i M t} \\
& \approx \frac{1}{\sqrt{2 V M}}\left(1-\frac{M^{2}}{2} t^{2}\right)-i \sqrt{\frac{M}{2 V}} t .
\end{aligned}
$$

Thus, the constants $B(M)$ and $C(M)$ in Eq. 120 are both $\sqrt{M V / 2}=O\left(M^{1 / 2}\right)$.
Next let us derive Eq. 129) for this special case. The zero-mode contribution to the FP-ghost propagator with the IR divergences regularized with small mass $M$ is

$$
\begin{aligned}
D^{(0) a b}\left(t, t^{\prime}\right)= & \frac{i}{2 M V} \delta^{a b}\left\{\theta\left(t-t^{\prime}\right) \exp \left[-i M\left(t-t^{\prime}\right)\right]\right. \\
& \left.+\theta\left(t^{\prime}-t\right) \exp \left[i M\left(t-t^{\prime}\right)\right]\right\} \\
= & \frac{i}{2 M V} \delta^{a b} \exp \left(-i M\left|t-t^{\prime}\right|\right) \\
\approx & \delta^{a b}\left(\frac{i}{2 M V}+\frac{1}{2 V}\left|t-t^{\prime}\right|\right)
\end{aligned}
$$

Then

$$
D_{\text {eff }}^{(0) a b}=\frac{1}{2 V} \delta^{a b}\left|t-t^{\prime}\right| .
$$

Hence

$$
\frac{\partial^{2}}{\partial t \partial t^{\prime}} D_{\mathrm{eff}}^{(0) a b}=-\frac{1}{V} \delta^{a b} \delta\left(t-t^{\prime}\right),
$$

which is Eq. (129).

Next, we show that the requirement of time-translation invariance of the vacuum state for the theory with nonzero gauge parameter $\xi$ in the Lagrangian density (45) leads to a divergent zero-mode two-point function [68. This is in contrast to the de Sitter case we study in the next Appendix.

Since the two-point function is diagonal in the Liealgebra space, we study it for the Abelian theory. For $\xi \neq 0$, the field equation with $q=0$ for the gauge field $A_{\mu}$ on the static flat torus is

$$
\partial^{\mu}\left(\partial_{\mu} A_{\nu}-\partial_{\nu} A_{\mu}\right)+\frac{1}{\xi} \partial_{\nu} \partial_{\mu} A^{\mu}=0 .
$$

With the assumption $A_{i}=0$ and that $\partial_{i} A_{0}=0, i=$ $1,2, \cdots, n-1$, we have $d^{2} A_{0} / d t^{2}=0$, which can be solved as

$$
A_{0(0)}(t)=\frac{1}{\sqrt{V}}(\hat{Q}+t \hat{P}),
$$

where $\hat{Q}$ and $\hat{P}$ are constant operators. The equal-time commutation relation between $A_{0}$ and $\dot{A}_{0}$ reads

$$
\left[A_{0}(t, \mathbf{x}), \dot{A}_{0}\left(t, \mathbf{x}^{\prime}\right)\right]=-i \xi \delta\left(\mathbf{x}, \mathbf{x}^{\prime}\right)
$$

By integrating over $\mathbf{x}$ and $\mathbf{x}^{\prime}$ we find

$$
\left[A_{0(0)}(t), \dot{A}_{0(0)}(t)\right]=-\frac{i \xi}{V} \text {. }
$$

This implies $[\hat{Q}, \hat{P}]=-i \xi$. It is clear from Eq. (E6) that, if we require that the perturbative vacuum state $|0\rangle$ be time-translation invariant, then $\hat{P}|0\rangle=0$. This means that $\left\langle 0\left|\left[A_{0(0)}(0)\right]^{2}\right| 0\right\rangle=V^{-1}\left\langle 0\left|\hat{Q}^{2}\right| 0\right\rangle=\infty$, which can be inferred from Heisenberg's uncertainty relation $\left\langle 0\left|\hat{Q}^{2}\right| 0\right\rangle\left\langle 0\left|\hat{P}^{2}\right| 0\right\rangle \geq \xi^{2} / 4$. For the Landau gauge with $\xi=0$ the field equations imply $\hat{P}=0$, and it is possible to require the condition $A_{0(0)}(t)|0\rangle=0$ as described in Sec. III. 


\section{Appendix F: The gauge-field zero-mode condition for the de Sitter case}

In this appendix we show that the condition $\varpi_{\tilde{B}(0)}|0\rangle=0$ is automatically satisfied by the perturbative Bunch-Davies vacuum state $|0\rangle$ in the Landau gauge. This means that there will be no need to remove the zeromode contribution from the gauge-field propagator by hand. We omit the Lie-algebra indices in this appendix as well.

We first note that, under the assumption that $A_{0}$ is space-independent and that $A_{i}=0$, the free-field equation with $\xi \neq 0$,

$$
\nabla^{\nu}\left(\nabla_{\nu} A_{\mu}-\nabla_{\mu} A_{\nu}\right)+\frac{1}{\xi} \nabla_{\mu} \nabla_{\nu} A^{\nu}=0,
$$

becomes

$$
\frac{1}{V(t)} \frac{d}{d t}\left[V(t) A_{0}(t)\right]=\text { const. }
$$

(Notice that $\nabla_{\nu} A_{\mu}-\nabla_{\mu} A_{\nu}=0$ under the assumptions we have made.) Thus, the general solution is a linear combination of $[V(t)]^{-1}$ and $[V(t)]^{-1} \int_{0}^{t} d t^{\prime} V\left(t^{\prime}\right)$. It can be shown that the space-independent positive-frequency solution, which is the coefficient of the annihilation operator, is proportional to $\nabla_{\mu} F_{0}$, where $F_{0}$ is the positivefrequency solution to the scalar field equation given by Eq. (17). Writing this solution as $\left(A_{0}, A_{i}\right)=(\Phi(t), 0)$ and provisionally normalizing $\Phi(t)$ by requiring

$$
i \int d \boldsymbol{\theta} \sqrt{|g|}\left[\Phi^{*}(t) \nabla^{0} \Phi(t)-\Phi(t) \nabla^{0} \Phi^{*}(t)\right]=1,
$$

we find

$$
\Phi(t)=\frac{1}{\sqrt{2 c_{0}} V(t)}\left[\int_{0}^{t} d t^{\prime} V\left(t^{\prime}\right)+i c_{0}\right] .
$$

Now, as in the previous Appendix the equal-time canonical commutation relations lead to

$$
\left[A_{0(0)}(t), \dot{A}_{0(0)}(t)\right]=-\frac{i \xi}{V(t)} \text {. }
$$

By writing

$$
A_{0(0)}(t)=\Phi(t) a+\Phi^{*}(t) a^{\dagger},
$$

where the perturbative vacuum state $|0\rangle$ satisfies $a|0\rangle=$ 0 , we obtain $\left[a, a^{\dagger}\right]=-\xi$. By expressing the annihilation operator $a$ in terms of $A_{0(0)}(t)$ we can write the condition $a|0\rangle=0$ as

$$
\left\{\Phi^{*}(t) \frac{d}{d t}\left[V(t) A_{0(0)}(t)\right]-\frac{V(t)}{\sqrt{2 c_{0}}} A_{0(0)}(t)\right\}|0\rangle=0
$$

for all $\xi \neq 0$. This condition is $\xi$-independent, and it is natural to require it for $\xi=0$ as well. Now, recall that $V(t) A_{0(0)}(t)=Q_{A}$ is a conserved Noether charge if $\xi=0$ [see Eq. [63]], which implies $d\left[V(t) A_{0(0)}(t)\right] / d t=0$. By using this operator equation in Eq. F7) we find, recalling that $A_{0(0)}=-\varpi_{\tilde{B}(0)}$, that $\varpi_{\tilde{B}(0)}|0\rangle=0$. (We note in passing that the conservation of $Q_{A}$ implies $a^{\dagger}=-a$.)

Now, we readily find

$$
\left\langle 0\left|\left[A_{0(0)}(0)\right]^{2}\right| 0\right\rangle=-\frac{\xi c_{0}}{2[V(0)]^{2}}
$$

from Eq. (F6) with

$$
\Phi(0)=i \sqrt{\frac{c_{0}}{2}} \frac{1}{V(0)}
$$

and the commutation relation $\left[a, a^{\dagger}\right]=-\xi$. In the rest of this appendix we show that Eq. F8 results from the known two-point functions for the massless vector field in the literature [35, 69 71] as a consistency check.

The two-point function given by Eq. (45) of Ref. [71] for $n \geq 4$ can be written as

$$
\begin{aligned}
\Delta_{\mu \nu^{\prime}}\left(x, x^{\prime}\right)= & \frac{n-2}{n-3} H^{-2} D_{M_{0}}(Z) \partial_{\mu} \partial_{\nu^{\prime}} Z \\
& +\frac{1}{n-3} H^{-2}\left[\frac{d}{d Z} D_{M_{0}}(Z)\right]\left(\partial_{\mu} Z\right)\left(\partial_{\nu^{\prime}} Z\right) \\
& +\left(\xi-\frac{n-1}{n-3}\right) \partial_{\mu} \partial_{\nu^{\prime}} \tilde{D}(Z), \quad(\mathrm{F} 10)
\end{aligned}
$$

where $M_{0}=\sqrt{n-2} H$. Here, the variable $Z$ is defined by

$$
\begin{aligned}
Z & =\cos H \mu\left(x, x^{\prime}\right) \\
& =-\sinh H t \sinh H t^{\prime}+\cosh H t \cosh H t^{\prime} \cos \chi\left(x, x^{\prime}\right),
\end{aligned}
$$

where $\mu\left(x, x^{\prime}\right)$ is the spacelike geodesic distance between the two points $x$ and $x^{\prime}$ and where $\chi\left(x, x^{\prime}\right)$ is the angle on $S^{n-1}$ between the space components of these two points. The function $D_{M}(Z)$ is the two-point function for the minimally coupled scalar field with mass $M$, and the function $\tilde{D}(Z)$ is defined by

$$
\tilde{D}(Z)=-\lim _{M \rightarrow 0^{+}} \frac{\partial}{\partial M^{2}}\left[D_{M}(Z)-D_{M}(-1)\right] .
$$

We have $\left.\partial_{t} Z\right|_{t=t^{\prime}=0}=0$ and $\left.\partial_{t} \partial_{t^{\prime}} Z\right|_{t=t^{\prime}=0}=-H^{2}$.

The normalized zero mode for the minimally coupled massless scalar field with mass $M_{0}$ can be found from Eq. (5) with $\ell=0$ and $\lambda=(n-3) / 2$ as

$$
\begin{aligned}
G_{0}(t)= & H^{\frac{n-2}{2}}\left[\frac{\Gamma(n-2)}{2}\right]^{1 / 2} \frac{1}{H^{\frac{n-1}{2}} \sqrt{V(0)}} \\
& \times(\cosh H t)^{-\frac{n-2}{2}} \mathrm{P}_{\frac{n-4}{2}}^{-\frac{n-2}{2}}(i \sinh H t),
\end{aligned}
$$

where $1 /\left[H^{\frac{n-1}{2}} \sqrt{V(0)}\right]$ is the spherical harmonic on $S^{n-1}$ with $\ell=0$. The zero-mode contribution to $D_{M_{0}}(Z)$ 
is $G_{0}(t) G_{0}^{*}\left(t^{\prime}\right)$ whereas that to $D_{M}(Z)$ with small $M$ is $F_{0}(t) F_{0}^{*}\left(t^{\prime}\right)$, where $F_{0}(t)$ is given by Eq. (17). Then

$$
\begin{aligned}
\left\langle 0\left|\left[A_{0(0)}(0)\right]^{2}\right| 0\right\rangle & \\
= & -\frac{n-2}{n-3}\left|G_{0}(0)\right|^{2} \\
& -\left.\left(\xi-\frac{n-1}{n-3}\right) \lim _{M \rightarrow 0^{+}} \frac{\partial}{\partial M^{2}}\left|\partial_{t} F_{0}(t)\right|^{2}\right|_{t=0} .
\end{aligned}
$$

Using 8.756.1 of Ref. 64,

$$
\mathrm{P}_{\nu}^{-\mu}(0)=\frac{2^{-\mu} \sqrt{\pi}}{\Gamma\left(\frac{\nu+\mu}{2}+1\right) \Gamma\left(\frac{-\nu+\mu+1}{2}\right)},
$$

and using the doubling formula A15, we find

$$
\left|G_{0}(0)\right|^{2}=\frac{(n-1) c_{0}}{2(n-2)[V(0)]^{2}},
$$

where the constant $c_{0}$ is given by Eq. 18. We also find

$$
\left.\lim _{M \rightarrow 0^{+}} \frac{\partial}{\partial M^{2}}\left|\partial_{t} F_{0}(t)\right|^{2}\right|_{t=0}=\frac{c_{0}}{2[V(0)]^{2}}
$$

By substituting Eqs. (F16) and (F17) into Eq. (F14) we obtain Eq. (F8) as expected. For $n=2$ and 3 one can verify Eq. (F8) by explicit integration over the space using the two-point function found in Appendix $\mathrm{C}$ of Ref. [71].
[1] D. Kazanas, Astrophys. J. 241, L59 (1980).

[2] K. Sato, Mon. Not. R. Astron. Soc. 195, 467 (1981).

[3] A. H. Guth, Phys. Rev. D 23, 347 (1981).

[4] A. D. Linde, Phys. Lett. 108B, 389 (1982).

[5] A. Albrecht and P. J. Steinhardt, Phys. Rev. Lett. 48, 1220 (1982).

[6] P. A. R. Ade et al. (BICEP2 Collaboration), Phys. Rev. Lett. 112, 241101 (2014).

[7] A. G. Riess, A. V. Filippenko, P. Challis, A. Clocchiatti, A. Diercks, P. M. Garnavich, R. L. Gilliland, C. J. Hogan, S. Jha, R. P. Kirshner, B. Leibundgut, M. M. Phillips, D. Reiss, B. P. Schmidt, R. A. Schommer, R. C. Smith, J. Spyromilio, C. Stubbs, N. B. Suntzeff and J. Tonry, Astron. J. 116, 1009 (1998).

[8] S. Perlmutter et al., Astrophys. J. 517, 565 (1999).

[9] A. Strominger, J. High Energy Phys. 10 (2001) 034.

[10] L. D. Faddeev and V. N. Popov, Phys. Lett. 25B, 29 (1967).

[11] L. H. Ford and L. Parker, Phys. Rev. D 16, 245 (1977).

[12] B. Allen, Phys. Rev. D 32, 3136 (1985).

[13] M. Faizal and A. Higuchi, Phys. Rev. D 78, 067502 (2008).

[14] K. Kirsten and J. Garriga, Phys. Rev. D 48, 567 (1993).

[15] A. Higuchi, J. Math. Phys. (N.Y.) (N.Y.) 28, 1553 (1987); 43, 6385(E) (2002).

[16] N. A. Chernikov and E. A. Tagirov, Ann. Inst. Henri Poincaré, A 9, 109 (1968).

[17] T. Bunch and P. Davies, Proc. R. Soc. A 360, 117 (1978).

[18] G. W. Gibbons and S. W. Hawking, Phys. Rev. D 15, 2738 (1977).

[19] B. Allen and A. Folacci, Phys. Rev. D 35, 3771 (1987).

[20] D. Marolf, Classical Quantum Gravity 12, 1199 (1995).

[21] A. Higuchi, Classical Quantum Gravity 8, 1983 (1991).

[22] A. Higuchi, Classical Quantum Gravity 8, 2023 (1991).

[23] N. P. Landsman, J. Geom. Phys. 15, 285 (1995).

[24] A. Ashtekar, J. Lewandowski, D. Marolf, J. Mourão and T. Thiemann, J. Math. Phys. (N.Y.) 36, 6456 (1995).

[25] T. Kugo and I. Ojima, Phys. Lett. 73B, 459 (1978).

[26] T. Kugo and I. Ojima, Prog. Theor. Phys. Suppl. 66, 1 (1979).
[27] N. Nakanishi, Prog. Theor. Phys. 35, 1111 (1966).

[28] B. Lautrup, Mat.Fys. Medd. - K. Dan. Vidensk. Selsk. 35, 1 (1967).

[29] C. Becchi, A. Rouet and R. Stora, Ann. Phys. (N.Y.) 98, 287 (1976).

[30] M. Z. Iofa and I. V. Tyutin, Theor. Math. Phys. 27, 316 (1976).

[31] G. Curci and R. Ferrari, Nuovo Cimento Soc. Ital. Fis. 32A, 151 (1976).

[32] I. Ojima, Prog. Theor. Phys. 64, 625 (1980).

[33] L. Baulieu and J. Thierry-Mieg, Nucl. Phys. B197, 477 (1982).

[34] I. Ojima, Nucl. Phys. B143, 340 (1978).

[35] B. Allen and T. Jacobson, Commun. Math. Phys. 103, 669 (1986).

[36] J. S. Schwinger, J. Math. Phys. (N.Y.) (N.Y.) 2, 407 (1961).

[37] L. V. Keldysh, Zh. Eksp. Teor. Fiz. 47, 1515 (1964).

[38] M. B. Fröb and A. Roura (private communication).

[39] P. Hájíček, "A new generating functional for expectation values of field operators" (unpublished).

[40] B. S. Kay, Commun. Math. Phys. 71, 29 (1980).

[41] R. D. Jordan, Phys. Rev. D 33, 444 (1986).

[42] E. Calzetta and B. L. Hu, Phys. Rev. D 35, 495 (1987).

[43] L. H. Ford and L. Parker, Phys. Rev. D 16, 1601 (1977).

[44] A. Higuchi, Nucl. Phys. B282, 397 (1987).

[45] B. Allen, Nucl. Phys. B287, 743 (1987).

[46] A. Higuchi and S. S. Kouris, Classical Quantum Gravity 17, 3077 (2000).

[47] A. Higuchi, D. Marolf and I. A. Morrison, Classical Quantum Gravity 28, 245012 (2011).

[48] S. W. Hawking, T. Hertog and N. Turok, Phys. Rev. D 62, 063502 (2000).

[49] A. Higuchi and R. H. Weeks, Classical Quantum Gravity 20, 3005 (2003).

[50] R. P. Bernar, L. C. B. Crispino and A. Higuchi, Phys. Rev. D 90, 024045 (2014).

[51] B. Allen and M. Turyn, Nucl. Phys. B292, 813 (1987).

[52] A. Higuchi and S. S. Kouris, Classical Quantum Gravity 18, 4317 (2001). 
[53] I. A. Morrison, arXiv:1302.1860.

[54] M. Faizal and A. Higuchi, Phys. Rev. D 85, 124021 (2012).

[55] S. P. Miao, N. C. Tsamis and R. P. Woodard, J. Math. Phys. (N.Y.) 52, 122301 (2011).

[56] P. Mora, N. C. Tsamis and R. P. Woodard, J. Math. Phys. (N.Y.) 53, 122502 (2012).

[57] C. J. Fewster and D. S. Hunt, Rev. Math. Phys. 25, 1330003 (2013).

[58] A. Higuchi, arXiv:1204.1684.

[59] S. S. Kouris, Classical Quantum Gravity 18, 4961 (2001); 29, 169501(E) (2012).

[60] P. J. Mora, N. C. Tsamis and R. P. Woodard, Phys. Rev. D 86, 084016 (2012).

[61] M. B. Fröb, ArXiv:1409.7964.

[62] N. C. Tsamis and R. P. Woodard, Class. Quant. Grav. 26, 105006 (2009).

[63] T. Tanaka and Y. Urakawa, Prog. Theor. Exp. Phys.
(2014) 073E01.

[64] I. S. Gradshteyn and I. M. Ryzhik, Table of Integrals, Series, and Products, , 7th ed. (Elsevier/Academic Press, Amsterdam, 2007).

[65] R. Arnowitt, S. Deser, and C.W. Misner, in Gravitation: An Introduction to Current Research, edited by L. Witten (Wiley, New York, 1962).

[66] F. A. Berezin, The Method of Second Quantization (Academic Press, New York, 1966).

[67] M. E. Peskin and D. V. Schroeder, An Introduction to Quantum Field Theory (Westview Press, Boulder, CO, 1995).

[68] S. P. Miao, N. C. Tsamis and R. P. Woodard, J. Math. Phys. (N.Y.) 50, 122502 (2009).

[69] N. C. Tsamis and R. P. Woodard, J. Math. Phys. (N.Y.) (N.Y.) 48, 052306 (2007).

[70] A. Youssef, Phys. Rev. Lett. 107, 021101 (2011).

[71] M. B. Fröb and A. Higuchi, J. Math. Phys. 55, 062301 (2014). 